\title{
Stringy Hidden Valleys
}

\author{
Mirjam Cvetič, ${ }^{1,2}$ James Halverson, ${ }^{1,3}$ and Hernan Piragua ${ }^{1}$ \\ 1 Department of Physics and Astronomy, \\ University of Pennsylvania, Philadelphia, PA 19104-6396, USA \\ 2 Center for Applied Mathematics and Theoretical Physics, \\ University of Maribor, Maribor, Slovenia \\ ${ }^{3}$ Kavli Institute for Theoretical Physics, \\ University of California, Santa Barbara, CA 93106-4030, USA
}

\begin{abstract}
We study gauge theories where quasi-hidden sectors are added to the MSSM for the sake of string consistency conditions which would otherwise not be satisfied. We focus on quiver gauge theories motivated by weakly coupled type II orientifold compactifications. Model independent features in this class include an anomalous $U(1)_{V}$ symmetry which protects messenger masses and has strong consequences for superpotential couplings, a rich phenomenology of heavy and light $Z^{\prime}$ bosons, and axionic couplings required for anomaly cancellation via the Green-Schwarz mechanism. We discuss possibilities for dark matter and supersymmetry breaking in light of these generic features. Dark matter is necessarily non-baryonic, though many dark matter candidates have weak interactions. Most models have a $U(1)_{V} Y Y$ anomaly whose cancellation requires couplings which allow for dark matter annihilation into photons through intermediate axions or anomalous $Z^{\prime}$ bosons, as in two recently proposed scenarios. There is often an additional non-anomalous $U(1)$ symmetry which can give rise to a Fayet-like model of metastable supersymmetry breaking. Breaking of supersymmetry via SQCD can also be realized and flavor masses are often protected. Natural possibilities for mediation include gauge mediation, $Z^{\prime}$ mediation, and D-instanton mediation, though it is not possible to realize minimal gauge mediation with messengers added for string consistency.
\end{abstract}




\section{Contents}

1 Introduction $\quad 2$

2 Basic Setup and Guiding Principles 4

2.1 Symmetries, Spectrum, and String Consistency 5

$\begin{array}{lll}2.2 & \text { Classifying Three-node MSSM Quivers } & 7\end{array}$

2.3 Adding Hidden Sectors for Consistency 9

2.3.1 "Hidden Hypercharge" Quivers 10

2.4 Fractionally Charged Massive Particles 12

$\begin{array}{ll}2.5 & \text { Comments on Fluxes and Consistency Conditions } \\ \end{array}$

3 General Structure of Low Energy Physics 13

3.1 Light Messengers and Constrained Superpotential Couplings 14

3.2 Anomalies, Required Axionic Couplings, and $Z^{\prime}$ Bosons 15

$\begin{array}{lll}3.2 .1 & \text { For All Stringy Hidden Valleys } & 15\end{array}$

$\begin{array}{lll}3.2 .2 & \text { For All Hidden Hypercharge Quivers } & 16\end{array}$

3.3 Dark Matter and a Possible Monochromatic $\gamma$-ray Line 18

3.3.1 Annihilation via Axionic Couplings and $Z^{\prime} Z_{\gamma}$ Vertices $\quad 19$

3.3.2 Messenger Dark Matter and $U(1)_{V} Y Y$ Anomalies 20

3.3.3 Hidden Sector Dark Matter $\quad 21$

3.4 Spontaneous Global Supersymmetry Breaking 22

3.4.1 Breaking Supersymmetry with SQCD 22

3.4.2 Breaking Supersymmetry via a Retrofitted Fayet Model 25

$\begin{array}{ll}\text { 3.4.3 Possibilities for Mediation } & 26\end{array}$

3.5 Implications of $Z^{\prime}$ Masses for Low Energy Physics 29

3.6 Generality of Results and Higher-Node Extensions 29

4 Example Quivers $\quad 30$

4.1 Hidden Extensions of the Madrid Embedding 30

4.1.1 Dark Matter Annihilation into Photons via an Intermediate $Z^{\prime} \quad 30$

4.1.2 Many Light $Z^{\prime}$ Bosons $\quad 32$

4.1.3 Dark Matter Annihilation and Axionic Couplings 32

4.2 Hidden Extensions of the Non-Madrid Embedding 33

4.2.1 Breaking SUSY with a Fayet Model $\quad 34$

$\begin{array}{lll}\text { 4.2.2 } & \text { Breaking SUSY with SQCD } & 35\end{array}$

5 Conclusions $\quad 36$ 


\section{Introduction}

With new experimental data arriving from the Large Hadron Collider and other experiments, recent model building efforts include the study of quasi-hidden gauge sectors which could be discovered in the near future. Such sectors are well motivated from top-down constructions, and it is possible that they interact weakly, but non-trivially, with the standard model. These include hidden valleys [1], Higgs portals (for example [2]), dark photons [3], and a variety of dark matter models [4]. Hidden valleys, in particular, are strongly motivated by works on string compactifications, such as $[5,6]$. However, the top-down input into these broad classes of effective field theories is that hidden extensions of the standard model frequently exist, and therefore they should be considered. It would be better, when possible, to have a guiding principle from top-down considerations which lead to precise gauge theoretic structures.

Gauge theories arising in string compactifications are constrained by consistency conditions. For example, the ten dimensional type I superstring is anomaly free only for gauge group $\operatorname{Spin}(32) / \mathbb{Z}_{2}$. Global consistency conditions can also require the presence of hidden sectors. In the heterotic $E_{8} \times E_{8}$ string, standard model sectors are typically realized in one $E_{8}$ factor, while the other $E_{8}$ factor can give rise to rich hidden sector physics which depend on consistency conditions on a holomorphic vector bundles. In type II compactifications, similar constraints are placed on the ranks of gauge groups by Ramond-Ramond tadpole cancellation. These constraints can descend to constraints on the chiral spectrum which are necessary for string consistency. The constraints on the chiral matter spectrum are not always equivalent to the cancellation of four-dimensional gauge anomalies. On one hand, string theory provides mechanisms for the cancellation of certain anomalies, and therefore does not place constraints on the chiral matter spectrum which ensure their absence. On the other hand, there exist constraints on the chiral matter spectrum of a gauge theory which are necessary for string consistency, but for which there is no known field theoretic analog. We refer to such constraints as "stringy" constraints.

It is possible to enumerate all realizations of the MSSM in certain classes of gauge theories motivated by string compactification. Many of these theories do not satisfy conditions necessary for string consistency, and therefore some augmentation is required. We focus exclusively on the possibility of adding hidden sectors, where messenger fields are chosen in a way that remedies visible sector inconsistencies. Doing so can lead to specific gauge theoretic structures which are generic across a broad class of models and have important consequences for low energy physics. Extensions of the MSSM with hidden sectors added for consistency form a proper subset of the broader class of hidden valley models. For brevity, will refer to a model in this class as a "stringy hidden valley."

In this paper we study a specific class of stringy hidden valleys. In a class of quiver gauge theories motivated by weakly coupled type II orientifold compactifications and their duals, nearly all MSSM quivers do not satisfy the constraints necessary for RamondRamond tadpole cancellation. The violation of these constraints is suggestive of matter fields that should be added to the theory, and we study the possibility that these fields are messengers to a hidden sector. We will find that this class of theories generically has 


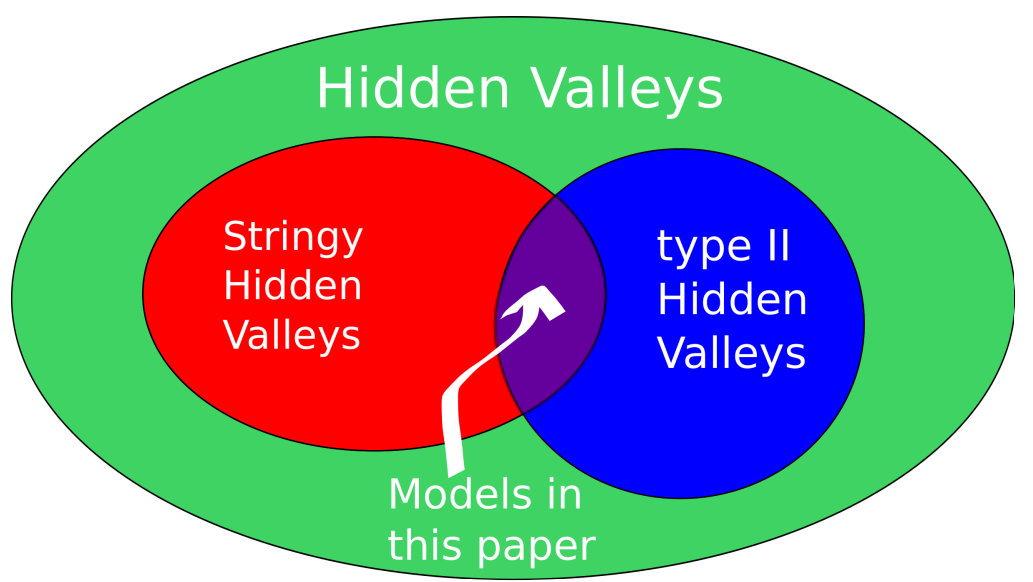

Figure 1. A depiction of different subsets of hidden valley models. The red region denotes those models where hidden extensions of the standard model are added for the sake of string consistency. The blue region denotes any hidden extension of a type II MSSM quiver. In this paper we study hidden extensions of type II MSSM quivers which are added for string consistency, denoted by the purple region.

messenger masses protected by symmetry, a rich structure of superpotential couplings, axionic couplings required for anomaly cancellation, and $Z^{\prime}$ bosons. These generic features give rise to interesting possibilities for supersymmetry breaking and dark matter, including annihilation processes with photons in the final state. We emphasize that these features are non-generic within the broad class of hidden valley models, but are generic in the class we study. We also emphasize that our results do not depend strongly on the number of nodes in the visible sector or possible flux contributions to tadpole cancellation conditions, as we will discuss. For brevity throughout, we will refer to these particular models as stringy hidden valleys, keeping in mind that they are a subset of stringy hidden valleys motivated by type II orientifold compactifications. See figure 1 .

Let us briefly describe the broader class of theories. Quiver gauge theories arising in weakly coupled type II orientifold compactifications ${ }^{1}$ and their duals have generic properties which affect low energy physics, including the presence of anomalous $U(1)$ symmetries which can forbid superpotential couplings in perturbation theory. These terms can be generated with suppression via D-instanton effects [10-12] or via couplings to singlets. Cancellation of $U(1)$ anomalies $^{2}$ via the Green-Schwarz mechanism requires the presence of Chern-Simons couplings of the form $B \wedge F$ and $\phi F \wedge F$, where the former gives a large Stückelberg mass to the associated $Z^{\prime}$ gauge boson. Consistent quivers must also satisfy conditions necessary for Ramond-Ramond tadpole cancellation. If they are to realize the MSSM, quivers must satisfy constraints necessary for the hypercharge to remain massless.

\footnotetext{
${ }^{1}$ Note that the quivers we consider differ slightly from those arising from D3-branes at singularities. There, the singularity structure determines the quiver. Here our approach is from the bottom-up: we build MSSM quivers and hidden sector extensions using the ingredients of type II string theory, independent of any global embedding or realization at a particular singularity. For D3-brane quivers, see for example [7-9] and references therein.

${ }^{2}$ This includes cubic abelian, mixed abelian non-abelian, and mixed abelian-gravitational anomalies.
} 
There has been much phenomenologically motivated work studying type II quiver gauge theories without hidden sectors. These studies have been both systematic and example-driven. For example, it has been shown [13-18] that D-instantons in MSSM quivers can give rise to realistic Yukawa couplings and neutrino masses while ensuring the absence of R-parity violating operators and dimension five proton decay operators. Alternative mechanisms for realistic neutrino masses have been proposed, including the generation of a realistic Dirac neutrino mass term [19] and a Weinberg operator $L H_{u} L H_{u}$ [20] by D-instantons. Other issues which have been studied include singlet-extended standard models [21], dynamical supersymmetry breaking [22], grand unification [23, 24], and $Z^{\prime}$ physics [25-27]. Systematics of hypercharge embeddings, bottom-up configurations, and global rational conformal field theory realizations have been carried out in [28].

This paper is very similar in spirit to [26], which also utilized string consistency conditions as the guiding principle for physics beyond the standard model. Since it is so closely related to this work, let us briefly review the conclusions. In [26], exotic matter was added to inconsistent type II MSSM quivers for the sake of consistency and in the most general way allowed by the construction, without adding additional quiver nodes. It was shown at the level of standard model representations that some exotics are much more likely than others, with a clear preference for MSSM singlets, $S U(2)_{L}$ triplets without hypercharge, and quasichiral pairs ${ }^{3}$. All of these possibilities could be relevant for LHC physics, as mass terms for the quasichiral pairs are forbidden by an anomalous $U(1)$ symmetry but could be generated at the $\mathrm{TeV}$ scale by string instantons or couplings to singlets. We also refer the reader to [26] for a more in depth discussion of the constraints we will import on quivers. In this paper, we will study the same class of MSSM quivers but will add hidden sectors for the sake of consistency.

This paper is organized as follows. In section 2 we review the structure of the quiver gauge theories we study, including string consistency conditions, and also the classification of three-node MSSM quivers. We introduce the possibility of adding hidden sectors only for the sake of string consistency and set notation used throughout. In section 3 we show that hidden sectors added for consistency have generic features which affect low energy physics, including an important symmetry $U(1)_{V}$, axionic couplings, and $Z^{\prime}$ bosons. These have important implications for various dark matter and supersymmetry breaking scenarios, which we discuss in detail. In section 4 we present explicit quivers realizing the general ideas of section 3 . In section 5 we conclude, briefly stating the main results and discussing possibilities for future work.

\section{Basic Setup and Guiding Principles}

In this section we will introduce a class of quiver gauge theories, emphasizing how they can arise in weakly coupled type II string compactifications. We discuss constraints on their chiral matter spectrum which are necessary for string consistency and introduce a guiding principle for adding hidden sectors. We will review the classification of three-node MSSM

\footnotetext{
${ }^{3}$ Quasichiral pairs are vector with respect to the standard model but chiral with respect to some other symmetry in the theory. In this case the symmetry is an anomalous $U(1)$.
} 
quivers. We will also discuss the applicability of our results to extensions of higher-node MSSM quivers.

\subsection{Symmetries, Spectrum, and String Consistency}

Let us introduce the class of theories we consider ${ }^{4}$, beginning with a discussion of symmetries. A compactification of weakly coupled type II string theory realizes gauge degrees of freedom as open strings ending on D-branes. For specificity, consider type IIa. A D6-brane wrapped on a generic three-cycle $\pi_{i}$ exhibits $U\left(N_{i}\right)$ gauge symmetry, though $S O\left(N_{i}\right)$ or $S p\left(N_{i}\right)$ gauge symmetry can exist if $\pi_{i}$ happens to be an orientifold invariant cycle. For generality and simplicity, we consider all gauge factors to be $U\left(N_{i}\right)$. In the presence of O6planes, every D6-brane has an associated orientifold-image brane, which will be important for the spectrum. We represent a brane together with its image brane as a node in a quiver, labeled by $U\left(N_{a}\right)$. The trace $U(1)$ of a $U\left(N_{i}\right)$ factor is often anomalous, and the associated abelian and mixed anomalies are automatically canceled in globally consistent string compactifications by four-dimensional terms coming from the dimensional reduction of the Chern-Simons D-brane action. In a bottom-up gauge theory, the necessary Chern-Simons terms can be added by hand (see [30], e.g.). In particular, anomaly cancellation requires terms of the form $\int d^{4} x B \wedge F$, which gives a string scale Stückelberg mass to the $U(1)$ with associated field strength $F$. Though these degrees of freedom can be integrated out at scales well below the associated $Z^{\prime}$ mass, the massive $U(1)$ 's impose global selection rules on the low energy effective action, forbidding many terms in the superpotential. We will realize the standard model gauge group via unitary factors $U(3)_{a} \times U(2)_{b} \times \prod_{\alpha=1}^{N} U(1)_{\alpha}$. We require the one linear combination $U(1)_{Y}=q_{a} U(1)_{a}+q_{b} U(1)_{b}+\sum_{\alpha=1}^{N} q_{\alpha} U(1)_{\alpha}$ is left massless $^{5}$ and can be identified as hypercharge. Such a combination is referred to as a hypercharge embedding. In addition, it is possible that another linear combination is left massless and is associated to a light $Z^{\prime}$ which must obtain a mass via the standard Higgs mechanism.

Let us discuss the spectrum. Chiral matter is localized at the intersection of two D6-branes and the chiral index is given by the topological intersection number of two three-cycles. Given a D6-brane on $\pi_{a}$, another D6-brane on $\pi_{b}$ and its image on $\pi_{b}^{\prime}$, strings localized at intersections of $\pi_{a}$ with $\pi_{b}$ carry representations $\left(N_{a}, \bar{N}_{b}\right)$ or $\left(\bar{N}_{a}, N_{b}\right)$ under $U\left(N_{a}\right) \times U\left(N_{b}\right)$ and strings localized at the intersections of $\pi_{a}$ and $\pi_{b}^{\prime}$ carry representations $\left(N_{a}, N_{b}\right)$ or $\left(\bar{N}_{a}, \bar{N}_{b}\right)$. The fundamental and antifundamental representations carry charge \pm 1 under the trace $U(1)$ 's, respectively. Therefore the branes can realize all four combinations of bifundamental representations, which we present as a bidirectional edge between the $N_{a}$ node and the $N_{b}$ node. String zero modes localized at the intersection of $\pi_{a}$ with $\pi_{a}^{\prime}$ come in symmetric or antisymmetric tensor representations under $U\left(N_{a}\right)$ and carry charge \pm 2 under the trace $U(1)_{a}$ associated to $U\left(N_{a}\right)$. We label these representations

\footnotetext{
${ }^{4}$ For a recent in-depth discussion of this class of theories, see [29].

${ }^{5}$ Throughout, we will use the phrases "massless U(1)" or "light $Z$ " to describe a $U(1)$ symmetry which is not required to obtain a Stückelberg mass. The terms "massive U(1)" or "heavy $Z^{\prime \prime}$ " will be used to denote a $U(1)$ symmetry which does not satisfy the constraints $(2.2)$ and therefore obtains a string scale Stückelberg mass.
} 


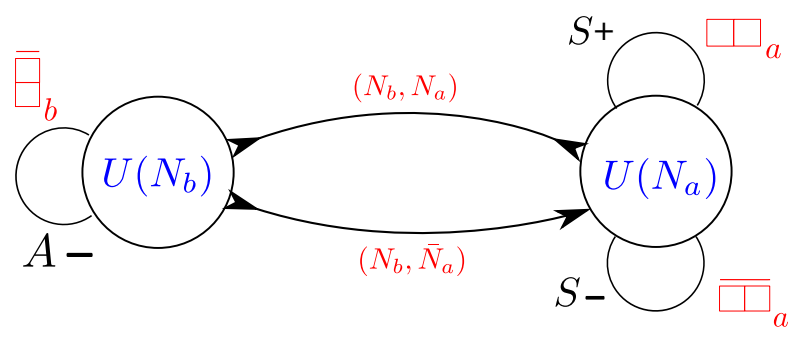

Figure 2. Example of a quiver. Each black line represents a field. The red symbols next to them are the associated representations, which we will henceforth omit since this data is equivalently communicated by the decorated edges. See the text for more details about this convention.

as an arrow from the $N_{a}$ node to itself with a + or - to denote the sign of the $U(1)$ charge and $S$ or $A$ to denote symmetric or antisymmetric. See figure 2 for an example. For a given set of nodes and hypercharge embedding, it is straightforward to enumerate all possible realizations of the MSSM spectrum.

We require that the chiral matter spectrum of the quiver satisfies two sets of conditions. The first set are those necessary for tadpole cancellation ${ }^{6}$, which are

$$
\begin{aligned}
& N_{a} \geq 2: \quad \# a-\# \bar{a}+\left(N_{a}+4\right)\left(\# \square \square_{a}-\# \bar{\square}_{a}\right)+\left(N_{a}-4\right)\left(\# \boxminus a-\# \overline{\Xi_{a}}\right)=0 \\
& N_{a}=1: \quad \# a-\# \bar{a}+\left(N_{a}+4\right)\left(\# \square_{a}-\# \bar{\square} \square_{a}\right)=0 \bmod 3,
\end{aligned}
$$

for each $U\left(N_{a}\right)$ node, where we have denoted the fundamental and antifundamental by $a$ and $\bar{a}$. A $U(1)$ defined by an arbitrary linear combination $\sum_{x} q_{x} U(1)_{x}$ will obtain a string scale Stückelberg mass unless the masslessness conditions

$N_{a} \geq 2:$

$-q_{a} N_{a}\left(\# \square_{a}-\# \bar{\square}_{a}+\# \square_{a}-\# \bar{\Xi}_{a}\right)+\sum_{x \neq a} q_{x} N_{x}(\#(a, \bar{x})+\#(\bar{a}, \bar{x})-\#(\bar{a}, x)-\#(a, x))=0$

$N_{a}=1:$

$-q_{a} \frac{\# a-\# \bar{a}+8\left(\# \square_{a}-\# \bar{\square}_{a}\right)}{3}+\sum_{x \neq a} q_{x} N_{x}(\#(a, \bar{x})+\#(\bar{a}, \bar{x})-\#(\bar{a}, x)-\#(a, x))=0$.

are satisfied. The second set of conditions we impose is that the hypercharge embedding satisfies these masslessness conditions. In a given quiver, there may also be other linear combinations which satisfy these equations, giving light $Z^{\prime}$ bosons.

Let us define some terminology that we will use throughout to discuss these conditions.

\footnotetext{
${ }^{6}$ Tadpole cancellation is the requirement that the net Ramond-Ramond (D-brane) charge is canceled on the internal space. It is necessary for the consistency of a globally defined string compactification. For example, in type IIa tadpole cancellation places a homological constraint on three-cycles wrapped by D6branes which can be shown to descend to the weaker conditions (2.1) on chiral matter. These conditions are necessary but not sufficient for D6-brane tadpole cancellation.
} 
For a $U\left(N_{a}\right)$ gauge node, we refer to the lefthand side of the conditions necessary for tadpole cancellation and a massless hypercharge boson as the "T-charge" $T_{a}$ and the "M-charge" $M_{a}$. In the three-node MSSM quivers we consider with $U(3)_{a} \times U(2)_{b} \times U(1)_{c}$, we will ensure that the T-charges $T_{a}, T_{b}$, and $T_{c}$ satisfy (2.1) and the M-charges $M_{a}, M_{b}$, and $M_{c}$ satisfy the equations (2.2). In addition, we may refer to the contributions of certain sets of fields to some T-charge or M-charge, where the context will make the content clear. For example, $T_{b}^{\text {mess }}$ could be the contribution of messenger fields to $T_{b}$.

The equations necessary for tadpole cancellation for $N_{a} \geq 3$ are the conditions necessary for the cancellation of cubic $S U\left(N_{a}\right)$ anomalies. It is crucial that consistent chiral spectra do not give rise to these anomalies, since the Green-Schwarz mechanism cannot cancel them. The corresponding field theory constraints do not exist for $N_{a}=2$ or $N_{a}=1$, however, and we refer to these as "stringy" constraints. We refer the reader to [26] for a recent in-depth discussion of these constraints and field theoretic constraints. These constraints are often violated for MSSM quivers. Our guiding principle will be to add hidden sectors so that they are satisfied.

\subsection{Classifying Three-node MSSM Quivers}

Our results regarding stringy hidden valleys will apply to essentially any MSSM quiver with non-zero T-charge which is canceled by the non-zero T-charge of messengers to a hidden sector. However, three-node MSSM quivers and their extensions provide an excellent example. Let us review their classification.

Consider a quiver with $U(3)_{a} \times U(2)_{b} \times U(1)_{c}$ gauge symmetry, which is the minimal number of nodes that can realize the MSSM gauge group and chiral spectrum at low energies. $S U(3) \times S U(2)_{L}$ of the standard model arise from the $U(3)$ and $U(2)$ factors and hypercharge must arise as a linear combination

$$
U(1)_{Y}=q_{a} U(1)_{a}+q_{b} U(1)_{b}+q_{c} U(1)_{c}
$$

of the trace $U(1)$ 's. There are only two possible sets $\left(q_{a}, q_{b}, q_{c}\right)$ which can realize the entire MSSM spectrum utilizing bifundamental, symmetric, and antisymmetric tensor representations. The first is the well-known Madrid embedding [31], given by

$$
U(1)_{Y}=\frac{1}{6} U(1)_{a}+\frac{1}{2} U(1)_{c}
$$

and possible MSSM field representations given by ${ }^{7}$

$$
\begin{array}{rll}
Q: & (a, b)(a, \bar{b}), & u^{c}:(\bar{a}, \bar{c}), \quad d^{c}: \Xi_{a}(\bar{a}, c), \\
L: & (b, \bar{c})(\bar{b}, \bar{c}), & e^{c}: \square_{c}, \\
H_{u}: & (b, c)(\bar{b}, c), & H_{d}:(b, \bar{c})(\bar{b}, \bar{c}),
\end{array}
$$

\footnotetext{
${ }^{7}$ To avoid unnecessary notation throughout, we make the definitions $u^{c} \equiv u_{L}^{c}, d^{c} \equiv d_{L}^{c}, e^{c} \equiv e_{L}^{c}$, and $\nu^{c} \equiv \nu_{L}^{c}$.
} 
where the unbarred and barred letters represent the fundamental and antifundamental representations of the associated nodes. MSSM singlets can be realized as $日_{b}$ or $\bar{\Xi}_{b}$, and for this embedding we define the chiral excess of singlets to be $N_{S} \equiv \# \mathrm{~B}_{b}-\# \bar{\Theta}_{b}$. Lacking a better name, the other hypercharge embedding is the non-Madrid embedding, given by

$$
U(1)_{Y}=-\frac{1}{3} U(1)_{a}-\frac{1}{2} U(1)_{b}
$$

and the possible MSSM field representations are given by

$$
\begin{aligned}
& Q:(a, \bar{b}), \quad u^{c}: \boxminus_{a}, \quad d^{c}:(\bar{a}, c)(\bar{a}, \bar{c}), \\
& L:(b, c)(b, \bar{c}), \quad e^{c}: \bar{\Xi}_{b}, \\
& H_{u}:(\bar{b}, c)(\bar{b}, \bar{c}), \quad H_{d}:(b, \bar{c})(b, \bar{c}) .
\end{aligned}
$$

MSSM singlets can be realized as $\square_{c}$ or $\bar{\square}_{c}$, and for this embedding we define the chiral excess of singlets to be $N_{S} \equiv \# \square \square_{c}-\# \square_{c}$. Depending on the coupling of these singlets to MSSM fields, they could be right-handed neutrinos $\nu^{c}$ or singlets $S$ which give rise to a dynamical $\mu$-term. See [21] for singlet-extended MSSM quivers in this class.

Given the possible MSSM field representations in the Madrid and non-Madrid embeddings, (2.5) and (2.7), it is possible to enumerate ${ }^{8}$ all three-node realizations of the exact MSSM spectrum. One can also compute the T-charges and M-charges from equations (2.1) and (2.2). The possible T-charges for the Madrid quivers are given by

$$
T_{a}=0 \quad T_{b}= \pm 2 n \quad T_{c}=0 \bmod 3 \quad \text { with } \quad n \in\{0, \ldots, 7\}
$$

and all M-charges are automatically zero. That is, all of the conditions necessary for a massless hypercharge are satisfied and the conditions necessary for tadpole cancellation are only violated on the $U(2)_{b}$ node. If a chiral excess $N_{S}$ of singlets are added to the theory, the only difference is $T_{b}= \pm 2 n-2 N_{S}$. Performing the same analysis for the non-Madrid embedding, one obtains

$$
T_{a}=0 \quad T_{b}=0 \quad T_{c}=\{0,1,2\} \bmod 3,
$$

and all M-charges zero. Therefore, for the non-Madrid embedding the only T-charge or M-charge violation is on the $U(1)_{c}$ node. If a chiral excess $N_{S}$ of singlets are added to the theory, the possible T-charges and M-charges remain the same but the multiplicity of quivers changes due to the new fields. For the Madrid embedding with $N_{S}=0$, there are 160 quivers in all, 144 of which violate the $T_{b}$ condition. For the non-Madrid embedding with $N_{S}=0$, there are 40 quivers in all, 24 of which violate the $T_{c}$ condition. With regards to anomalies, a simple calculation shows that 144 of the 160 Madrid quivers have a $U(1)_{b} Y Y$ anomaly, and all non-zero anomaly coefficients are non-integral. All 40 quivers with the non-Madrid quivers have a $U(1)_{c} Y Y$ anomaly, and all anomaly coefficients are

\footnotetext{
${ }^{8}$ Since $L$ and $H_{d}$ carry the same standard model quantum numbers, we treat them as one field with multiplicity four in our counting.
} 
non-integral. We will utilize these facts later when discussing hidden sectors.

It is remarkable that most three-node MSSM quivers violate the conditions necessary for tadpole cancellation. This is also true of MSSM quivers with a larger number of nodes. Let us briefly discuss possible field theoretic explanations in terms of anomalies. Since the spectrum under $S U(3) \times S U(2)_{L} \times U(1)_{Y}$ of each quiver is the exact MSSM, perhaps with singlets added, there are no cubic non-abelian anomalies and there is no global $S U(2)$ anomaly [32]. Any mixed anomalies involving abelian symmetries, such as the $U(1)_{b} Y Y$ and $U(1)_{c} Y Y$ anomalies just discussed, can be canceled by the introduction of appropriate Chern-Simons terms ${ }^{9}$, and therefore these quivers are consistent quantum field theories. However, without further modification any quiver which does not satisfy the conditions necessary for tadpole cancellation cannot be embedded into the types of string compactifications we have discussed. One possible solution is to add matter to the theory so that the inconsistent quivers become consistent. This was done for three-node quivers in [26], as discussed in the introduction, where it was shown that string consistency conditions prefer some standard model representations over others. We now turn to another possible solution.

\subsection{Adding Hidden Sectors for Consistency}

In section 2.2, we showed that most three-node quivers with the exact MSSM spectrum are not consistent with conditions necessary for tadpole cancellation, despite being consistent as quantum field theories after the addition of Chern-Simons terms. Higher node MSSM quivers also exhibit this behavior. We discussed the possibility of adding matter to the visible sector nodes to cancel the T-charge contribution of MSSM fields.

Another possible solution is to add hidden gauge sectors where bifundamental messenger fields with one end on a visible sector node $V$ cancel any overshooting in T-charge or M-charge, ensuring that hidden sector is also consistent. This will be our guiding principle for physics beyond the standard model. We call ${ }^{10}$ any hidden sector of this type a "stringy hidden valley." The setup is heuristically depicted in figure 3 and looks similar to common depictions of hidden sectors. We have a visible sector with fields transforming under $S U(3) \times S U(2)_{L} \times U(1)_{Y}$ and associated anomalous $U(1)$ 's, a hidden sector with fields transforming under some hidden sector gauge group $G_{H}$, and messenger fields transforming under both the visible sector group and $G_{H}$. However, the setups we study will differ from generic hidden sectors in at least two important ways. First, since our hidden sectors are added to cancel some visible sector $T_{V}$-charge, the messenger fields to the $V$ node will always vector-like with respect to the standard model but chiral ${ }^{11}$ under $U(1)_{V}$, and therefore their masses are always protected. Phenomenologically this very important, since pairs which are vector with respect to all symmetries have string scale masses at a

\footnotetext{
${ }^{9}$ Consistent type II string compactifications provide these terms automatically via dimensional reduction of the Chern-Simons D-brane action. See [30], for example, for a similar discussion purely in field theory.

${ }^{10} \mathrm{As}$ emphasized in the introduction, stringy hidden valleys are potentially a much broader class than the models studied here. More specifically, we are studying stringy hidden valleys in type II quivers.

${ }^{11}$ We will refer to such fields as "quasichiral".
} 


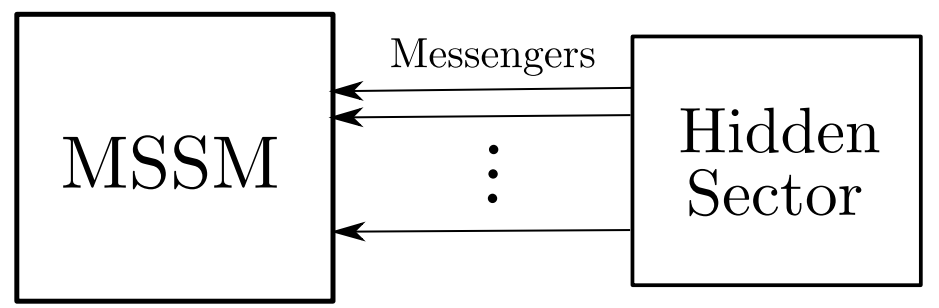

Figure 3. Heuristic depiction of the stringy hidden valleys studied in this paper. The setup is similar to standard hidden sector setups, but the messengers are chiral under an anomalous $U(1)_{V}$ symmetry in the visible sector in order to cancel the non-zero $T_{V}$ charge of MSSM fields.

generic point in the moduli space of a string compactification. Second, the structure of the hidden sectors will be constrained by string consistency conditions.

In the three-node Madrid and non-Madrid embeddings string consistency requires that messengers are added to the $U(2)_{b}$ and $U(1)_{c}$ nodes, respectively, and therefore the symmetry $U(1)_{V}$ which charges the messengers are the trace $U(1)_{b}$ and $U(1)_{c}$. In discussing $U(1)_{V}$, though, we will be able to address aspects of low energy physics in these models which do not depend on the visible sector hypercharge embedding.

There are two basic types of nodes in a hidden sector: those connected directly to the visible sector via messengers and those which are not. We utilize lower case latin indices for the first type, calling them $H_{i}$ nodes, and capital latin indices for the second type, calling them $H_{I}$ nodes. The most general hidden sector could be composed of $n$ connected graphs, each disjoint from one another but connected to the visible sector via messengers. When differentiating between disconnected clusters in the hidden sector, we will utilize a superscripted $(m)$ to describe quantities in the $m^{\text {th }}$ cluster. For example, $H_{I}^{(m)}$ nodes would be nodes in the $m^{\text {th }}$ cluster which are not directly connected to the visible sector.

\subsection{1 "Hidden Hypercharge" Quivers}

In adding a hidden sector, it is possible that the hypercharge embedding is modified due to contributions from trace $U(1)$ 's of hidden sector nodes. Before adding hidden sectors, the MSSM quivers had a hypercharge embedding given by a linear combination of trace $U(1)$ 's of visible sector nodes. We previously called this $U(1)_{Y}$, but henceforth will call it $U(1)_{Y, V}$. We write the full hypercharge embedding as a linear combination

$$
U(1)_{Y}=U(1)_{Y, V}+U(1)_{Y, H},
$$

where $U(1)_{Y, H}$ is the contribution from hidden sector nodes. While it may seem strange to discuss hidden sector contributions to hypercharge, it is possible to nevertheless ensure that all hidden sector fields are MSSM singlets, and thus we should consider this possibility. We will argue in a moment that it is actually generic to have $U(1)_{Y, H}$ non-trivial. We call any quiver with a non-trivial $U(1)_{Y, H}$ a "hidden hypercharge" quiver. We call its hidden sector a "hypercharged stringy hidden valley".

Any hidden hypercharge quiver is very constrained. Consider the possibility of a single- 
cluster hidden sector with non-trivial $U(1)_{Y . H}$. In general, we could have

$$
U(1)_{Y, H}=\sum_{\alpha} q_{\alpha} U(1)_{\alpha}
$$

where the sum is over all hidden sector nodes indexed by $\alpha$. For the cluster to be connected and hidden, however, every hidden sector node must be connected to another hidden sector node by a field which is an MSSM singlet. This requires $\left|q_{\alpha}\right|=\left|q_{\beta}\right|$ for all $\alpha$ and $\beta$ where the type of bifundamental is dictated by the signs of $q_{\alpha}$. We take $q_{\alpha}=q_{\beta} \equiv q$ for all $\alpha, \beta$ without loss of generality, which requires that we use only bifundamentals $(\alpha, \bar{\beta})$ and $(\bar{\alpha}, \beta)$ and not $(\alpha, \beta)$ or $(\bar{\alpha}, \bar{\beta})$. Thus, the non-trivial contribution to hypercharge "propagates" through the entire cluster by the requirement that bifundamental fields are MSSM singlets. For an $n$-cluster hidden sector, then, we have ${ }^{12}$

$$
U(1)_{Y, H}=\sum_{m=1}^{n} U(1)_{Y, H}^{(m)} \quad \text { where } \quad U(1)_{Y, H}^{(m)} \equiv q^{(m)} \sum_{\alpha} U(1)_{\alpha}^{(m)} \equiv q^{(m)} U(1)_{F}^{(m)}
$$

and the first sum is over the $n$ clusters while the second is over all nodes in the $m^{\text {th }}$ cluster. The key observation is that all nodes in a given cluster contribute to the hypercharge embedding in the same way. Thus, any cluster is labeled by a rational number $q^{(m)}$ which determines its contribution to hypercharge. Physically, $q^{(m)}$ has a simple interpretation: $\pm q^{(m)}$ is the hypercharge of the messengers to the $m^{\text {th }}$ hidden cluster.

At the level of graph theory, a hidden cluster with $q^{(m)} \neq 0$ is a directed graph: any nonmessenger edge is between two hidden nodes and has its two arrows in the same direction, in which case we may draw a single arrow indicating the direction. Any loop in hidden sector edges is directed, in contrast with the general case, and the corresponding fields give a perturbative superpotential coupling composed of MSSM singlets. For example, consider figure 4. The messengers are the fields between nodes of type $H_{i}$ and node $V$, which has an anomalous $U(1)_{V}$ that charges the messengers. The hidden sector fields are fields connecting hidden sector nodes. Letting $S_{i j}$ be the singlet from nodes $i$ to $j$, one perturbative superpotential coupling in this quiver is given by $\frac{1}{M_{s}} S_{1, n+1} S_{n+1, n+3} S_{n+3,2} S_{2,1}$, represented by the closed loop between those nodes.

Since the notion of a hidden hypercharge quiver may still seem strange, let us make some comments regarding generality. We have argued that the requirement that hidden sector fields are MSSM singlets fixes the hypercharge contribution of the $m^{\text {th }}$ hidden cluster up to a single number $q^{(m)}$. For an $n$ cluster hidden sector, the contribution to the hypercharge is determined by the tuple $\left(q^{(1)}, q^{(2)}, \ldots, q^{(n)}\right)$. Only one possibility, given by the tuple $(0, \ldots, 0)$, has a trivial contribution to hypercharge. Any other tuple gives a hidden hypercharge quiver. Though hidden hypercharge quivers are not required by string consistency, it would introduce a loss of generality to not consider them. Furthermore, we will argue in section 2.4 that hidden sector contributions to the hypercharge embedding

\footnotetext{
${ }^{12}$ We have defined $U(1)_{F}^{(m)}$ for later convenience, since it is a particularly natural linear combination to consider. We will see it can play a role in both supersymmetry breaking and stabilization of messenger dark matter.
} 


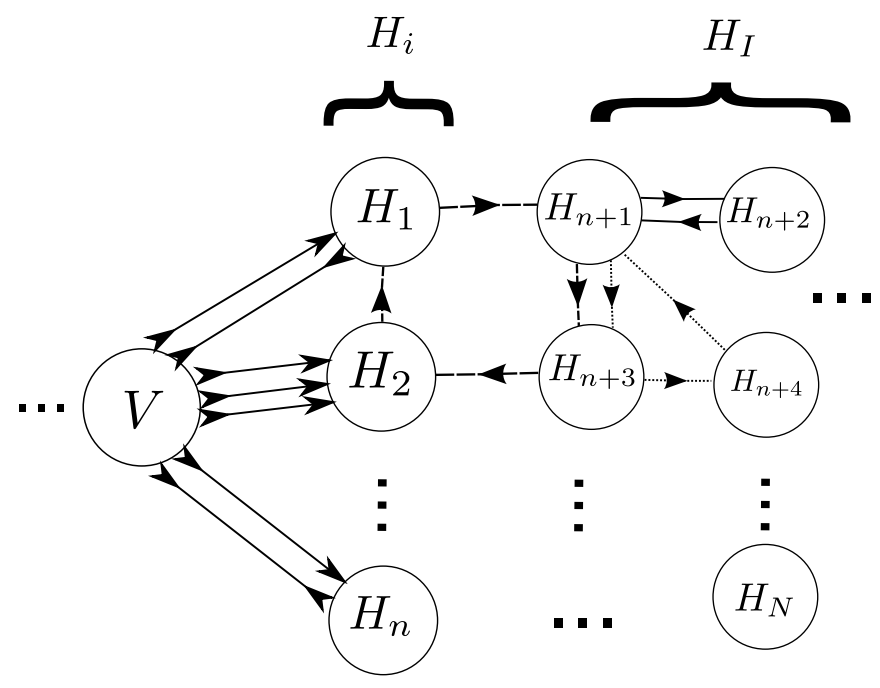

Figure 4. An example of a hidden sector. The first column of nodes, $H_{i}$, are connected to the visible sector node $V$ through the messengers. The nodes on the right $H_{I}$ do not have messengers attached. Closed loops, such as the ones given by the dotted and dashed lines, give perturbative superpotential couplings. We have not labeled the gauge groups to emphasize the general structure. However, $T_{H_{2}} \neq 0$ in this quiver requires that it is a $U(1)$ node. $H_{1}$ could be non-abelian.

are often required to avoid messenger fields with fractional electric charge.

\subsection{Fractionally Charged Massive Particles}

In this brief subsection we will address an important aspect of phenomenology which must be taken into account when building quivers with hidden sectors.

Globally consistent string compactifications and quivers in the class we study often exhibit particles with fractional electric charge ${ }^{13}$. If they exist, the lightest fractionally charged massive particle is stable and its relic density is subject to strong constraints from primordial nucleosynthesis and the cosmic microwave background. Recent work [33] shows that their existence is essentially ruled out. Therefore, we do not consider quivers which give rise to particles with fractional electric charge. This is an important phenomenological consideration which greatly constrains the quivers we study. For example, in extensions of the Madrid embedding any cluster with a $U(1) H_{i}$ type node must have $q^{(m)} \in \mathbb{Z}+\frac{1}{2}$, since the messengers are necessarily doublets of $S U(2)_{L}$ and otherwise they would have fractional electric charge. In non-Madrid extensions, the the messengers are necessarily singlets of $S U(3)_{Q C D} \times S U(2)_{L}$ and therefore any cluster with a $U(1) H_{i}$ type node must have $q^{(m)} \in \mathbb{Z}$. In a cluster with only non-abelian $H_{i}$ type nodes, confinement can relax these constraints, though others exist which depend on the rank of the non-abelian gauge nodes.

\footnotetext{
${ }^{13}$ Standard model quarks have fractional electric charge, but this does not pose an issue since they are bound into mesons and baryons, which have integral electric charge.
} 


\subsection{Comments on Fluxes and Consistency Conditions}

In the type IIb string, fluxes are crucial for moduli stabilization and can contribute to tadpole cancellation conditions. This is well known for the D3-tadpole, for example. Since the string consistency conditions (2.1) are necessary for tadpole cancellation, let us consider how the conditions may change in the presence of fluxes.

We will work in type IIa, since the consistency conditions are easily derived there. In the absence of fluxes, D6-brane tadpole cancellation gives a homological constraint on the three-cycles wrapped by D6-branes and O6-planes,

$$
\sum_{b} N_{b}\left(\pi_{b}+\pi_{b^{\prime}}\right)=4 \pi_{O 6}
$$

The conditions (2.1) arise from intersecting this equation with each cycle $\pi_{\alpha}$ on which a D6-brane stack is wrapped and utilizing the relations between topological intersection numbers and chiral indices. See [29] for more details. The equations (2.1) can only be altered if (2.13) is altered. Schematically ${ }^{14}$ this must be of the form

$$
\pi_{\text {flux }}+\sum_{b} N_{b}\left(\pi_{b}+\pi_{b^{\prime}}\right)=4 \pi_{O 6}
$$

Intersecting with $\pi_{\alpha}$ gives a set of constraints similar (2.1), except for an additional possible contribution $T_{\alpha}^{\text {flux }} \equiv \pi_{\alpha} \cdot \pi_{\text {flux }}$. If $T_{\alpha}^{\text {flux }}=0$, the constraint on $T_{\alpha}$ is unchanged. $T_{\alpha}^{\text {flux }} \neq 0$ the equations can be altered, though the flux must be tuned if it is to precisely cancel any net T-charge of an MSSM quiver. In the generic case the flux contribution will not exactly cancel the net T-charge of the MSSM quiver, and additional matter must still be added for the sake of consistency.

Therefore, the addition of fluxes will not significantly alter the physical conclusions of this paper, which rely entirely on the fact that there is some net T-charge which is canceled by quasichiral messengers to a hidden sector.

\section{General Structure of Low Energy Physics}

Equipped with a guiding principle for adding hidden sectors, it is possible to make statements about low energy physics which are true of the stringy hidden valleys we consider, but not of a generic hidden valley. The major conclusions in this section will not require the specification of the visible sector matter content, the hidden sector matter content, or the hypercharge embedding. This is because stringy hidden valleys generically exhibit symmetries and chiral spectra which are non-generic within the class of all hidden valleys. Presentation of concrete quivers will be saved for section 4 .

We will begin with a discussion of the symmetry $U(1)_{V}$ which charges the messengers and the associated implications for superpotential couplings. Certain classes of couplings are forbidden in perturbation theory; others are always highly suppressed. We will then turn to a discussion of anomalies, phenomenologically relevant axionic couplings necessary

\footnotetext{
${ }^{14}$ See $[34,35]$ for a detailed discussion of possible flux contributions to the D6-brane tadpole.
} 
for their cancellation, and $Z^{\prime}$ physics. We will show that these basic building blocks lead to interesting models of dark matter and supersymmetry breaking, realizing mechanisms already present in the literature.

\subsection{Light Messengers and Constrained Superpotential Couplings}

One type of symmetry plays a distinguished role in all stringy hidden valleys. Messengers are added with a net excess of $T_{V}$ charge for some visible sector node $V$, in which case they are chiral under $U(1)_{V}$. In extensions of three-node quivers with the Madrid or non-Madrid hypercharge embedding the node $V$ is the $U(2)_{b}$ node or the $U(1)_{c}$ node, respectively, in which case messengers are chiral under $U(1)_{b}$ or $U(1)_{c}$. In the case of multiple visible sector nodes $V$ which charge messengers of different type, there will be more than one symmetry $U(1)_{V}$. We emphasize that the conclusions of this section are generic for the models we study.

Let us discuss possible superpotential couplings, beginning with couplings present in perturbation theory. Label a generic superpotential coupling of $i$ chiral supermultiplets in the visible, messenger, and hidden sectors as $\mathcal{O}_{V}^{i}, \mathcal{O}_{M}^{i}$, and $\mathcal{O}_{H}^{i}$, respectively. Depending on the fields present in the coupling, it is possible that $\mathcal{O}_{V}^{i}$ and $\mathcal{O}_{H}^{i}$ are present in perturbation theory. However, messenger fields carry anomalous $U(1)$ charge of the same sign and therefore no operator $\mathcal{O}_{M}^{i}$ is present in perturbation theory. This is particularly important for the messenger mass terms $\mathcal{O}_{M}^{2}$, since the anomalous $U(1)$ symmetry prevents the associated fields from acquiring a string scale mass and decoupling from low energy physics. On general grounds, therefore, the messenger mass is always protected by symmetry and could be generated at the TeV scale via instantons or couplings to singlet VEVs. This is certainly not required in a generic hidden valley or hidden sector, and it will have important consequences.

Symmetries also dictate the structure of mixed couplings $\mathcal{O}_{V}^{i} \mathcal{O}_{M}^{j} \mathcal{O}_{H}^{k}$. Since messenger fields are the only fields transforming under both visible sector and hidden sector gauge nodes, there are no closed paths in the quiver corresponding to couplings of the form $\mathcal{O}_{V}^{i} \mathcal{O}_{H}^{j}$, and thus these operators are not present in perturbation theory. Couplings of the form $\mathcal{O}_{V}^{i} \mathcal{O}_{M}^{1} \mathcal{O}_{H}^{j}$ are forbidden since they carry always anomalous $U(1)$ charge. Thus, the lowest dimension perturbative superpotential coupling involving both visible sector and hidden sector fields is a non-renormalizable term $\sim \frac{1}{M_{s}} \mathcal{O}_{V}^{1} \mathcal{O}_{M}^{2} \mathcal{O}_{H}^{1}$. It is also possible to couple messenger fields only to visible sector fields, and the lowest dimension couplings of this type are $\sim \mathcal{O}_{V}^{1} \mathcal{O}_{M}^{2}$. Couplings of messengers to hidden sector fields carry $U(1)_{V}$ charge and are forbidden. We have exhausted the possibilities for couplings present in perturbation theory.

Let us discuss couplings not present in perturbation theory, due to carrying anomalous $U(1)$ charge. These coupling can be generated non-perturbatively via $\mathrm{D}$-instantons, in which case they are exponentially suppressed by a factor $e^{-T}$, a suppression factor dependent upon the volume of the cycle wrapped by the instanton. A perturbatively forbidden coupling $\mathcal{O}$ can also be generated from a perturbative couplings to singlets $\mathcal{O}_{S} \sim S^{n} \mathcal{O}$ if the singlet has a vacuum expectation value. Compared to the possibility of obtaining $\mathcal{O}$ directly in perturbation theory, obtaining it via couplings to singlets suppress $\mathcal{O}$ by a factor 


\begin{tabular}{c|ccc|} 
Coupling Structure & Perturbative & Instanton-induced & Singlet Couplings \\
\hline $\mathcal{O}_{V}^{i}$ & $M_{s}^{3-i}$ & $e^{-T} M_{s}^{3-i}$ & $M_{s}^{3-(n+i)}$ \\
\hline $\mathcal{O}_{M}^{i}$ & Forbidden & $e^{-T} M_{s}^{3-i}$ & $M_{s}^{3-(n+i)}$ \\
\hline $\mathcal{O}_{H}^{i}$ & $M_{s}^{3-i}$ & $e^{-T} M_{s}^{3-i}$ & $M_{s}^{3-(n+i)}$ \\
\hline $\mathcal{O}_{V}^{i} \mathcal{O}_{M}^{j} \mathcal{O}_{H}^{k}$ & $M_{s}^{3-(i+j+k)}, j \geq 2$ & $e^{-T} M_{s}^{3-(i+j+k)}$ & $M_{s}^{3-(i+j+k+n)}$ \\
\hline $\mathcal{O}_{V}^{i} \mathcal{O}_{M}^{j}$ & $M_{s}^{3-(i+j)}, j \geq 2$ & $e^{-T} M_{s}^{3-(i+j)}$ & $M_{s}^{3-(i+j+n)}$ \\
\hline $\mathcal{O}_{M}^{i} \mathcal{O}_{H}^{j}$ & Forbidden & $e^{-T} M_{s}^{3-(i+j)}$ & $M_{s}^{3-(i+j+n)}$ \\
\hline $\mathcal{O}_{V}^{i} \mathcal{O}_{H}^{j}$ & Forbidden & $e^{-T} M_{s}^{3-(i+j)}$ & Forbidden \\
\hline
\end{tabular}

Table 1. Couplings and their respective suppressions. All indices are $\geq 1$. In the last column $n$ is the power of the singlet that acquires the VEV. In extensions of an MSSM quiver with the Madrid embedding there is extra suppression, since messengers transform under $S U(2)_{L}$ and gauge invariance requires at least $\mathcal{O}_{M}^{2}$ for couplings of type $\mathcal{O}_{M}^{i}$ and $\mathcal{O}_{M}^{i} \mathcal{O}_{H}^{j}$ obtained via instantons or singlet couplings. If the visible sector realizes the exact MSSM spectrum, then some couplings will be further suppressed by the requirement of MSSM gauge invariance. For example $\mathcal{O}_{V}^{i}$ and $\mathcal{O}_{V}^{i} \mathcal{O}_{M}^{j}$ would have $i \geq 2$.

of $\left(\langle S\rangle / M_{s}\right)^{n}$. In either case, the coupling $\mathcal{O}$ receives a large suppression, which could ${ }^{15}$ easily be $10^{-14}$. The results for the minimum suppression of a coupling $\mathcal{O}$ are presented in table 1, where we have utilized MSSM gauge invariance and the chirality of messengers under $U(1)_{V}$ to determine the minimum suppression for each coupling. For simplicity, we also require that singlets which generate couplings are not messengers, since then $\mathcal{O}_{V} \mathcal{O}_{H}$ couplings could arise from $\mathcal{O}_{V} \mathcal{O}_{M} \mathcal{O}_{H}$ couplings, for example. Relaxing this assumption would complicate the analysis without significantly changing the structure of couplings.

In summary, the structure of superpotential couplings is strongly constrained by symmetries generically present in stringy hidden valleys. The most important observation compared to a generic quiver is that $\mathcal{O}_{M}^{i}$ and $\mathcal{O}_{M}^{i} \mathcal{O}_{H}^{j}$ couplings are forbidden in perturbation theory, and thus are very suppressed if they are present at all. Suppression of these and other couplings play an important role in ensuring that the hidden sector doesn't couple strongly to visible sector fields, despite being present in the same connected quiver.

\subsection{Anomalies, Required Axionic Couplings, and $Z^{\prime}$ Bosons}

All quivers in the broad class motivated by type II orientifold compactifications have a rich structure of anomalies, axionic couplings required for their cancellation, and $Z^{\prime}$ physics. In this section we will make statements about them which are generic for stringy hidden valleys, but not for the broader class. We will see that these features can have important consequences for dark matter and supersymmetry breaking.

\subsubsection{For All Stringy Hidden Valleys}

We have emphasized that all stringy hidden sectors have messengers which are chiral under an anomalous $U(1)$ symmetry $U(1)_{V}$. Therefore there is a $U(1)_{V} U(1)_{i} U(1)_{i}$ anomaly for

\footnotetext{
${ }^{15}$ See [19], for example, where instantons were used to generate Dirac neutrino masses of the observed order without the seesaw mechanism.
} 
the $U(1)$ of any $H_{i}$ node with $U\left(N_{i}\right)$ gauge symmetry. In addition, if $N_{i}>2$, there are mixed $U(1)_{V} S U\left(N_{i}\right)^{2}$ anomalies.

These anomalies must be canceled via the Green-Schwarz mechanism through the introduction of Chern-Simons terms. The mixed abelian anomalies are canceled by the introduction of terms of the form

$$
\int d^{4} x B_{V} \wedge F_{V} \quad \text { and } \quad \int d^{4} x \phi_{V} F_{i} \wedge F_{i}
$$

where $F_{V}$ and $F_{i}$ are the field strengths of $U(1)_{V}$ and $U(1)_{i}, B_{V}$ is the two-form which gives a Stückelberg mass to $U(1)_{V}$, and $\phi_{V}$ is the zero-form which is the four-dimensional Hodge dual of $B_{V}$, i.e. $d B_{V}=*_{4 d} d \phi_{V}$. If $N_{i}>1$, the mixed abelian non-abelian anomalies require terms of the form

$$
\int d^{4} x \phi_{V} \operatorname{Tr}\left(G_{i} \wedge G_{i}\right)
$$

where $G_{i}$ is the field strength of $S U\left(N_{i}\right)$. These conclusions hold for any stringy hidden valley, and the axionic terms can play an important phenomenological role.

Since the coupling $\int B_{V} \wedge F_{V}$ is always present, $U(1)_{V}$ is always a massive $U(1) . U(1)_{V}$ gauge interactions are suppressed via the large $Z_{V}^{\prime}$ mass. This has strong implications for interactions between messengers and visible sector particles charged under $U(1)_{V}$. See section 3.5 for a discussion of $Z^{\prime}$ masses and low energy physics.

Finally, a non-generic but common possibility is to have a chiral excess of messengers ${ }^{16}$ on the $H_{i}$ node. In this case there is a $U(1)_{i} U(1)_{V} U(1)_{V}$ anomaly, whose cancellation requires terms of the form $\int B_{i} \wedge F_{i}$ and $\int \phi_{i} F_{V} \wedge F_{V}$ and $U(1)_{i}$ is a massive $U(1)$. If there is not an excess of chiral messengers on $H_{i}$, it is possible that $U(1)_{i}$ is massless, but this cannot be determined without further specification of the hidden sector spectrum.

\subsubsection{For All Hidden Hypercharge Quivers}

In this section we discuss further aspects of anomalies, axionic couplings, and $Z^{\prime}$ physics which are true of any hidden hypercharge quiver. The additional physics is governed by the fact that the hidden sector contributes non-trivially to the hypercharge embedding, which will have interesting implications for dark matter annihilation processes with photons in the final state.

\section{$U(1)_{V} Y Y$ Anomalies and Axionic Couplings}

Let us first consider $U(1)_{V} Y Y$ anomalies. Since messengers carry hypercharge and are chiral under $U(1)_{V}$, they will always contribute to this anomaly. Unlike $U(1)_{V} U(1)_{i} U(1)_{i}$ anomalies, $U(1)_{V} Y Y$ anomalies can also receive contributions from visible sector fields, in which case it may be possible that visible sector and messenger contributions cancel and there is no anomaly. We will now argue that this is almost never the case for extensions of three-node quivers. The only possible loophole is in the case where confinement relaxes constraints on $q^{(m)}$ necessary to ensure the absence of fractionally charged massive particles.

\footnotetext{
${ }^{16}$ By this we simply mean that the net $U(1){ }_{i}$ charge of the messengers is non-zero.
} 
Let us begin with the Madrid embedding where $U(1)_{V}=U(1)_{b}$, taking $k$ messengers. As discussed in section 2.2 all but sixteen of the three-node MSSM quivers have a $U(1)_{b} Y Y$ anomaly, and any non-zero anomaly coefficient is non-integral. The $k$ messengers are $S U(2)_{L}$ doublets and give a net contribution to the $U(1)_{b} Y Y$ anomaly of $\sum_{m}-2 k\left(q^{(m)}\right)^{2}$. We must have $q^{(m)}=\frac{N_{m}}{2}$ with $N_{m} \in \mathbb{Z}$ for either the absence of fractionally charged massive particles ${ }^{17}$ or the existence of an electrically neutral messenger component, and in this case, since cancellation of $T_{b}$ charge requires $k$ even, the messengers give an integral contribution to the $U(1)_{b} Y Y$ anomaly coefficient. Since the contribution of all possible visible sectors to the anomaly coefficient is non-integral, all stringy hidden valley extensions of three-node MSSM quivers with the Madrid embedding and $q^{(m)}=\frac{N_{m}}{2}$ exhibit a $U(1)_{b} Y Y$ anomaly. For non-Madrid extensions, we will often require $q^{(m)} \in \mathbb{Z}$ to ensure the absence of fractionally charged massive particles, in which case messengers will give an integral contribution to the $U(1)_{c} Y Y$ anomaly which will not cancel the non-integral contribution from visible sector fields. Thus, all non-Madrid quivers of this type have a $U(1)_{c} Y Y$ anomaly. Summarizing, any extensions of a Madrid (non-Madrid) quiver with $q^{(m)} \in \mathbb{Z}+\frac{1}{2}$ $\left(q^{(m)} \in \mathbb{Z}\right)$ has a $U(1)_{b} Y Y\left(U(1)_{c} Y Y\right)$ anomaly.

We have just argued that $U(1)_{V} Y Y$ anomalies are extremely common in hidden hypercharge quivers, being very precise with the case of extensions of visible sectors with three-nodes. For any quiver with such an anomaly, anomaly cancellation via the GreenSchwarz mechanism requires the presence of a term

$$
\int d^{4} x \phi_{V} F_{Y} \wedge F_{Y}
$$

where $F_{Y}$ is the field strength of hypercharge. In section 3.3 will see that these terms play a crucial role in recent models for dark matter annihilation processes with photons in the final state.

\section{The $Z^{\prime}$ Physics of Hidden Hypercharge Quivers}

$Z^{\prime}$ bosons appear in many top-down constructions and can greatly impact low energy physics, as reviewed in [36]. The models we have proposed have a rich structure of $Z^{\prime}$ physics. In hidden hypercharge quivers there is a "natural" $U(1)$ which is usually massless, and is always massless for any extension of a three-node quiver. Recall from section 2.3 that we write the hypercharge embedding as

$$
U(1)_{Y}=U(1)_{Y, V}+U(1)_{Y, H}
$$

where the two terms are the contributions of the visible sector and the hidden sector to the hypercharge linear combination, respectively. For all quivers, we require that the linear combination $U(1)_{Y}$ satisfies the linear equations (2.2). If $U(1)_{Y, V}$ independently satisfies these linear equations, as it does for all extensions of three-node MSSM quivers ${ }^{18}$, then

\footnotetext{
${ }^{17}$ In a cluster with a $U(1)$ node. As mentioned in section 2.4 this constraint can be relaxed if all nodes in the cluster $m$ are non-abelian.

${ }^{18} U(1)_{Y, V}$ is just the Madrid or non-Madrid linear combination. These linear combinations are massless
} 
$U(1)_{Y, H}$ will also satisfy these equations. Therefore any such quiver will give rise to a light $Z^{\prime}$ boson. We will think of this $Z^{\prime}$ as coming from $U(1)_{Y, H}$ and henceforth call it $Z_{Y, H}^{\prime}$, though we could equivalently consider $U(1)_{Y, V}$. $Z_{Y, H}^{\prime}$ couples to any messengers which have hypercharge, but never to hidden sector fields. $U(1)_{Y, H}$ is closely related to $U(1)_{F}$. If there is a single cluster, $U(1)_{Y, H}$ is just $U(1)_{F}$ rescaled by $q \equiv q^{(m)}$.

There are further interesting statements that one can make about $Z^{\prime}$ physics in hidden hypercharge quivers. To do so, it is useful to consider two possibilities for the cluster $m$ : the case where cluster $m$ has a chiral excess of messengers on some node $H_{i}^{(m)}$, and the case where it has no such chiral excess for any $H_{i}^{(m)}$ node.

Let us first consider the possibility where there is no chiral excess of messengers on any $H_{i}^{(m)}$ node, and examine the linear combination $U(1)_{Y, H}^{(m)}$ as in equation $(2.12)$. From (2.2) the conditions on a node $H_{i}^{(m)}$ necessary for $U(1)_{Y, H}^{(m)}$ to remain massless are

$$
q^{(m)} \sum_{\alpha} N_{\alpha}[\#(\bar{V}, \bar{\alpha})-\#(\bar{V}, \alpha)]=0
$$

where the sum is over hidden sector nodes in cluster $m$ and we remind the reader that messengers are chiral under $U(1)_{V}$. This is equivalent to the condition on $H_{i}^{(m)}$ nodes necessary for a massless $U(1)_{Y, H}$, and therefore they are satisfied since $U(1)_{Y, H}$ is massless. Similar statements apply for $H_{I}^{(m)}$ nodes. The only condition left to satisfy is the condition on the $V$ node, given by

$$
q^{(m)} \sum_{i} N_{i}[\#(\bar{V}, \bar{i})-\#(\bar{V}, i)]=0 .
$$

This is stronger than the $V$ node condition for a necessary $U(1)_{Y, H}$, but it is satisfied since we are considering the case where there is no chiral excess of messengers on any $H_{i}^{(m)}$ node, so that each term in square brackets is zero. Therefore $U(1)_{Y, H}^{(m)}$ is also massless and there is yet another light $Z^{\prime}$. If there are many such clusters, there can be many light $Z^{\prime}$ bosons.

Let us consider the other case, where there is a chiral excess of messengers on some node $H_{i}^{(m)}$. From (3.5) it is clear there is not necessarily a light $Z^{\prime}$ corresponding to $U(1)_{Y, H}^{(m)}$. However, the chiral excess induces $U(1)_{i} Y Y$ mixed abelian anomaly since the messengers ending on node $i$ also carry hypercharge. Such an anomaly is canceled by Chern-Simons terms of the form $\int d^{4} x B_{i} \wedge F_{i}$ and $\int d^{4} x \phi_{i} F_{Y} \wedge F_{Y}$ where $F_{i}$ and $F_{Y}$ are the field strengths of $U(1)_{i}$ and $U(1)_{Y}$ and $B_{i}$ and $\phi_{i}$ are a two-form and its Hodge dual zero-form. The Chern-Simons terms introduced to cancel anomalies of this type can play an important role in dark matter annihilation, as we will now discuss.

\subsection{Dark Matter and a Possible Monochromatic $\gamma$-ray Line}

It has been known for many years that string consistency often requires the presence of hidden sectors which can give rise to interesting dark matter candidates. In the $E_{8} \times E_{8}$ heterotic string, the standard model spectrum is typically constructed from one of the

for all three-node MSSM quivers, and it is easy to see that adding a hidden sector will not cause these conditions to be violated for $U(1)_{Y, V}$. 
two $E_{8}$ factors. The other factor generically gives rise to another gauge sector which interacts with the visible sector only gravitationally. In weakly coupled type II orientifold compactifications and F-theory, "filler branes" which do not intersect the standard model branes are often required for tadpole cancellation. See, for example, [37]. These interact gravitationally with the standard mode, but not via gauge interactions.

It is also possible that nature contains a dark matter sector which couples weakly to the standard model, but nevertheless can exhibit dark matter annihilation into standard model particles via gauge interactions or suppressed couplings to visible sector particles. In the last six months many models of this type have been explored, due in part to the possible experimental observation of a $\gamma$-ray line from dark matter annihilation near the galactic center [38-41]. Regardless of whether this signal survives further scrutiny, particularly by the Fermi LAT collaboration itself, it is important to discuss whether dark matter candidates in our theories can annihilate via processes with visible sector particles in the final state, particularly photons.

\subsubsection{Annihilation via Axionic Couplings and $Z^{\prime} Z_{\gamma}$ Vertices}

We showed in section 3.2 that the stringy hidden valleys we study generically have a rich structure of $U(1)$ physics and axionic couplings. These can have important consequences for dark matter annihilation.

Let us briefly review two ideas in the literature which are very common in our models and give rise to dark matter annihilation processes with photons in the final state. The first utilizes an intermediate anomalous $Z^{\prime}$ boson to give the dark matter annihilation process $\chi \bar{\chi} \rightarrow Z^{\prime} \rightarrow Z \gamma$. This was proposed a few years ago in [42] and more recently in [43] after the possible observation of the $\gamma$-line. The key feature is an anomalous $U(1)$ symmetry under which dark matter is charged. Anomaly cancellation via the GreenSchwarz mechanism requires the presence of axionic couplings which give an effective $Z^{\prime} Z \gamma$ vertex that makes the annihilation process possible. One difficulty is that the annihilation cross section is suppressed by the $Z^{\prime}$ mass, which is typically very large. See section 3.5.

This possibility is extremely common in our models. Structurally, all that is needed is dark matter charged under some symmetry $U(1)_{X}$ and a $U(1)_{X} Y Y$ anomaly. In our models there are many $U(1)$ symmetries which may play this role and this possibility could be checked on a quiver by quiver basis. However, $U(1)_{V}$ is a distinguished $U(1)$ symmetry in all of our quivers. As we have argued in section 3.2, hidden hypercharge quivers always have messengers which contribute to the $U(1)_{V} Y Y$ anomaly coefficient and the quiver exhibit a $U(1)_{V} Y Y$ anomaly unless the contribution from the visible sector precisely cancels those of the messengers. We have argued that this never happens for extensions of three-node MSSM quivers, and therefore a $U(1)_{V} Y Y$ anomaly is generic in those models. In addition, even if the hidden sector is not hypercharged there is almost always a $U(1)_{V} Y Y$ anomaly just from the visible sector contribution. Thus, dark matter charged under $U(1)_{V}$ can nearly always realize the scenario of [42], at least structurally. By the definition of $U(1)_{V}$, such dark matter is messenger dark matter, which we will discuss.

Another possibility was recently proposed [44] which utilized similar axionic couplings. The theory has a hidden sector with a non-anomalous $U(1)_{X}$ and an $S U(N)$ gauge factor 
with quarks carrying appropriate $U(1)_{X}$ charge to give rise to neutral or $U(1)_{X}$-charged hidden sector pions. There are axionic couplings of the form $\phi F_{Y} \wedge F_{Y}$ and $\phi G \wedge G$ where $F_{Y}$ and $G$ are the hypercharge and $S U(N)$ field strengths, respectively. The $U(1)_{X}$-charged pions are stable due to being the lightest $U(1)_{X}$ charged particles and are identified as dark matter. They can annihilate to $U(1)_{X}$-neutral hidden sector pions which can then decay to photons via the axionic couplings. See section 4.1.3 for a concrete realization similar to this possibility in a stringy hidden valley.

Our models frequently realize axionic couplings similar to these. In certain cases it is possible to add these axionic couplings by hand, as in [44]. The more interesting case, however, is when they are required for anomaly cancellation. As argued in section 3.2, there is a $U(1)_{V} U(1)_{i}^{2}$ anomaly for any $H_{i}$ node and also a $U(1)_{V} S U\left(N_{i}\right)^{2}$ if $H_{i}$ is nonabelian, requiring the presence of couplings $\phi_{V} F_{i} \wedge F_{i}$ and $\phi_{V} G_{i} \wedge G_{i}$. The key coupling allowing annihilation to photons is the axionic coupling to the hypercharge field strength, here $\phi_{V} F_{Y} \wedge F_{Y}$. This is necessary for the cancellation of a $U(1)_{V} Y Y$ anomaly, which nearly always exists. Therefore our models typically have the couplings utilized in necessary to explain dark matter annihilation via the mechanism of [44], or a similar mechanism. In a given quiver, there may be anomalous $U(1)$ 's other than $U(1)_{V}$ which could play this role.

\subsubsection{Messenger Dark Matter and $U(1)_{V} Y Y$ Anomalies}

Since all quivers we study have messenger fields to hidden gauge nodes, one simple possibility is that dark matter is comprised of messengers fields $M$ and $\tilde{M}$. Since they are quasichiral, the messenger mass is always protected by symmetry and can therefore be light, perhaps $\mathcal{O}(\mathrm{GeV})$ or $\mathcal{O}(\mathrm{TeV})$. We see from table 1 that any perturbative superpotential coupling of messengers to a standard model field is string suppressed, and that similar couplings obtained via instanton effects or couplings to singlets are also very suppressed. Messenger dark matter in stringy hidden valleys will always be non-baryonic, since string consistency does not require the addition of messengers charged under $S U(3)_{Q C D}$ when extending MSSM quivers.

Let us discuss possibilities under which messenger dark matter is stable against decay. A simple possibility is that a symmetry ensures stability, which is certainly possible if there is a natural symmetry under which only messengers are charged. As shown in section 3.2, quivers with a hypercharged stringy hidden sector very frequently ${ }^{19}$ have a massless $U(1)_{Y, H}$ which charges only the messengers and could protect messenger dark matter candidates from decay. In addition, any hidden hypercharge quiver and many others will have $U(1)_{F}^{(m)}$ symmetries, perhaps anomalous, which charge only the messengers to the $m^{\text {th }}$ hidden cluster. In concrete quivers, there could be other massless $U(1)$ symmetries which charge the messengers, or massive $U(1)$ symmetries. Therefore, symmetries which could protect messenger dark matter from decay are very common.

Let us discuss possible annihilation processes for messenger dark matter in generality. $U(1)_{V}$ always charges both the messengers and some set of standard model fields, allowing for dark matter annihilation via $\chi \bar{\chi} \rightarrow Z_{V}^{\prime} \rightarrow f \bar{f}$ for standard model fermions $f$. In

\footnotetext{
${ }^{19}$ Always, for extensions of three-node quivers.
} 
addition, unless visible sector contributions to the $U(1)_{V} Y Y$ anomaly coefficient exactly cancel the messenger contributions, dark matter can annihilate to photons via $\chi \bar{\chi} \rightarrow Z_{V}^{\prime} \rightarrow$ $Z \gamma$ as discussed in section 3.3.1. However, $Z_{V}^{\prime}$ is heavy and dark matter annihilation cross sections are suppressed. Purely in a low energy effective theory, though, one can treat the mass of $Z_{V}^{\prime}$ as a parameter and constrain the phenomenologically allowed parameter space, as in [43]. See section 3.5 for a discussion of anomalous $Z^{\prime}$ masses. In addition, any stringy hidden valley necessarily gives rise to couplings $\phi_{V} F_{i} \wedge F_{i}$ and $\phi_{V} G_{i} \wedge G_{i}$. Since messengers end on $H_{i}$ nodes, the axionic couplings could give rise to dark matter annihilation processes with photons in the final state, similar to [44].

Let us discuss more specific possibilities which depend on the visible sector hypercharge embedding. For messenger dark matter to have any hope of being realistic in an extension of the Madrid embedding, it must be a messenger to a cluster with $q^{(m)}=\frac{1}{2}$, which is required for the $S U(2)_{L}$ charged messenger to have an electrically neutral component $\chi$. Such a particle is a natural WIMP candidate. For the Madrid embedding, $U(1)_{V}=U(1)_{b}$, and dark matter can annihilate into an anomalous $Z_{b}^{\prime}$. Since messengers are doublets of $S U(2)_{L}$, annihilation to $f \bar{f}$ via the process $\chi \bar{\chi} \rightarrow Z \rightarrow f \bar{f}$ will dominate over the process involving an intermediate $Z_{V}^{\prime}$. In an extension of the non-Madrid embedding, messenger fields must end on a cluster with $q^{(m)}=0$ for field to have an electrically neutral component $\chi$ and $U(1)_{V}=U(1)_{c}$. Dark matter can annihilate to $f \bar{f}$ via an intermediate anomalous $Z_{c}^{\prime}$. The messengers do not carry hypercharge, but in the case where the standard model fields generate a $U(1)_{c} Y Y$ anomaly, dark matter can nevertheless decay as $\chi \bar{\chi} \rightarrow Z_{c}^{\prime} \rightarrow Z \gamma$. This is possible for any extension of a three-node quiver, since there is always a $U(1)_{c} Y Y$ anomaly, as argued in section 3.2.

\subsubsection{Hidden Sector Dark Matter}

Another possibility is that dark matter is comprised of fields transforming only under hidden sector nodes. As such, they necessarily standard model singlets. Since hidden sector fields are much less constrained than messenger fields, there are more possibilities and we will therefore be brief. Symmetries ensuring stability are similar to the messenger dark matter case, except that hidden sector dark matter is not charged under $U(1)_{Y, H}$, the distinguished massless $U(1)$ common in hidden hypercharge quivers.

Since hidden sector dark matter does not carry $U(1)_{V}$ charge, it cannot decay via a $Z_{V}^{\prime} Z \gamma$ vertex. However, as argued in section 3.2 there are broad classes of quivers which exhibit a $U(1)_{i} Y Y$ anomaly, which introduces a $Z_{i}^{\prime} Z \gamma$ vertex into the theory, allowing for dark matter annihilation into photons via $Z_{i}^{\prime}$. In such a case dark matter is necessarily charged under $U\left(N_{i}\right)$ and could annihilate to photons via the axionic couplings $\phi_{V} F_{i} \wedge F_{i}$ and $\phi_{V} G_{i} \wedge G_{i}$ as suggested in [44]. This mechanism does not rely on the propagation of a heavy $Z_{i}^{\prime}$. Finally, there are never $U(1)_{I} Y Y$ anomalies, since this would require hidden sector fields which carry hypercharge. Therefore the $Z_{I}^{\prime} Z \gamma$ vertex is not required to exist in the low energy theory and it is unlikely ${ }^{20}$ that hidden sector dark matter ending only on $H_{I}$ nodes will decay into photons.

\footnotetext{
${ }^{20}$ In the absence of $B_{I} \wedge F_{I}$ couplings there could be $\phi_{I} F_{Y} \wedge F_{Y}$ and no $U(1)_{I} Y Y$ anomaly.
} 


\subsection{Spontaneous Global Supersymmetry Breaking}

In a globally consistent string compactification, the proper framework for discussing supersymmetry and its breaking is $\mathcal{N}=1$ supergravity, where the dynamics and stabilization of closed string moduli play an important role in determining possible supersymmetry breaking and mediation scenarios. As discussed, string consistency often requires the presence strongly coupled gauge sectors which interact only gravitationally with the standard model. It is possible that supersymmetry is broken in this sector and gravity mediation ensues. Such analyses require the specification of a global string compactification with moduli stabilized and is outside the realm of the quiver gauge theories we study. However, in the $M_{p l} \rightarrow \infty$ limit it is natural to study the possibility of global supersymmetry breaking. Though an embedding into supergravity may spoil ${ }^{21}$ the global supersymmetry conclusions gained via studying a quiver gauge theory, this is the best one can do at the quiver level and the conclusions may nevertheless hold in supergravity embeddings. In this section we will discussed global supersymmetry breaking scenarios in stringy hidden valleys.

One way to break supersymmetry is to embed a non-abelian gauge theory into the low energy spectrum which exhibits strong gauge dynamics that break supersymmetry [45]. A prototype which has been studied extensively is $\mathcal{N}=1$ supersymmetric QCD with $S U\left(N_{c}\right)$ gauge symmetry and $N_{f}$ vector-like flavors [46]. Metastable supersymmetry breaking $[47,48]$ is a common and intriguing possibility, in SQCD and in general. In addition, classic supersymmetry breaking models which do not utilize strong gauge dynamics have been realized in simple D-brane quivers [12, 49], where D-instantons play a crucial role in determining scales in the model. Global realizations include [50, 51]. We find that supersymmetry breaking via SQCD and a retrofitted Fayet model similar to those of [49] can appear naturally in the models we study.

One important feature that we must consider with either SQCD or Fayet breaking is that messenger fields often play a crucial role. In such a case supersymmetry breaking can give vacuum expectation values to the scalar components of the messengers, breaking the MSSM gauge group in the common case of non-singlet messengers. In particular, in extensions of the Madrid embedding the messengers carry $S U(2)_{L}$ charge and supersymmetry breaking involving messengers VEVs would trigger electroweak symmetry breaking. For simplicity we will avoid this possibility, when necessary, in the examples of section 4 .

\subsubsection{Breaking Supersymmetry with SQCD}

Since we take hidden sector gauge group $G_{H}=\prod_{i} U\left(N_{i}\right)$, realizations of supersymmetry breaking with strong gauge dynamics necessarily require an $U\left(N_{i}\right)$ gauge group. In a generic hidden sector there could be many such factors with rich gauge dynamics, but for simplicity we will restrict our attention to the possibility of a single non-abelian factor with gauge group $U\left(N_{c}\right)$ with $N_{f}$ flavors which are vector-like with respect to $U\left(N_{c}\right)$. All flavors are necessarily bifundamentals, and for simplicity we also require that they have one end on a common node which is not the $U\left(N_{c}\right)$ node. Given these restrictions, it

\footnotetext{
${ }^{21}$ For example, in a string compactification the Fayet-Iliopoulos term $\xi$ depends on closed string moduli and may dynamically relax to zero, restoring supersymmetry in the Fayet models we will discuss. Realizing this model in supergravity would require stabilization at a point in moduli space with non-zero $\xi$.
} 
is natural to classify the possibilities according to whether the flavors are messengers or hidden sector fields. We refer to these scenarios as "messenger SQCD" and "hidden sector SQCD", respectively. Of course, hybrid scenarios are also possible if $U\left(N_{c}\right)$ is an $H_{i}$ node.

Over time it has been shown that SQCD can break supersymmetry for many values of $N_{f}$ and $N_{c}$, originally in the confined $N_{f}<N_{c}$ regime in [46]. More recently it has been shown $[47,48]$ that SQCD can give rise to metastable supersymmetry breaking in the free magnetic range $N_{c}+1 \leq N_{f}<\frac{3}{2} N_{c}$. For a recent discussion of these ideas and their history, see [52].

\section{Messenger Flavors}

If the $U\left(N_{c}\right)$ node is an $H_{i}$ node, the SQCD flavors can end on a visible sector node $V$ with $U\left(N_{V}\right)$ gauge symmetry and $N_{V} \in\{1,2\}$. The flavors are what we have been calling "messenger" fields, where this should not necessarily be confused with messengers of gauge mediated supersymmetry breaking. The quiver takes the form shown in figure 5 and the

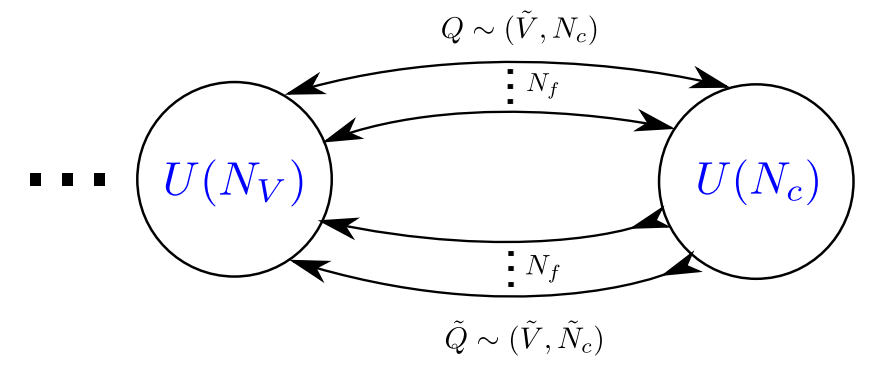

Figure 5. SQCD with messenger flavors. In each node the gauge group is showed in blue. $U\left(N_{V}\right)$ is the visible sector node, and $U\left(N_{c}\right)$ is an $H_{i}$ type hidden sector node. The messenger sector is made of $N_{f}$ copies of $Q$ and $\tilde{Q}$.

field content beyond the standard model is $N_{f}$ copies of $Q \sim\left(\bar{V}, N_{c}\right)$ and $\tilde{Q} \sim\left(\bar{V}, \bar{N}_{c}\right)$, and the flavors are chiral with respect to the trace $U(1)_{V}$ of $U\left(N_{V}\right)$. To avoid detailed analyses of supersymmetry breaking scenarios for different values of $N_{f}$ and $N_{c}$, we will utilize facts about $S U\left(N_{c}\right)$ SQCD despite the fact that our gauge group is $U\left(N_{c}\right)$. This is certainly a valid assumption at scales below the mass of the non-anomalous $Z^{\prime}$ boson associated to the trace $U(1)$ of $U\left(N_{c}\right)$. We will give a concrete example of these models in section 4.2. Let us discuss some generic features here.

An important feature of these realizations of SQCD is that the mass term $Q \tilde{Q}$ is protected by symmetry but can be generated at a low scale via D-brane instantons or couplings to singlets. In the absence of this symmetry, the flavors will typically obtain a large mass far above the confinement scale $\Lambda_{N_{c}}$, giving a pure SQCD theory at low energies which does not break supersymmetry. We view this as an advantage of these models and assume that the masses of the flavors is far below the confinement scale. A natural concern in this theory is that it may be difficult to realize the Affleck-Dine-Seiberg non-perturbative superpotential which plays an important role in supersymmetry breaking, since $Q \tilde{Q}$ explicitly appears and is forbidden by symmetry. However, it is known [53] that 
a gauge invariant ${ }^{22} \mathrm{ADS}$ superpotential can be generated even in the case where $Q \tilde{Q}$ carries net anomalous $U(1)_{V}$ charge. We have argued in section 3.2 that $U(1)_{V}$ is always anomalous in models with stringy hidden sectors. Given these arguments, one can apply standard techniques of supersymmetry breaking via SQCD with various various of $N_{f}$ and $N_{c}$.

For SQCD with messenger flavors in our models, the allowed values of $N_{f}$ and $N_{c}$ are constrained by the fact that messengers are added to cancel some non-zero T-charge, and the T-charges are concretely determined by possible visible sector realizations of the MSSM. For example, in extensions of the three-node Madrid hypercharge embedding the only possible non-zero T-charge is $T_{b}= \pm 2 n$ for $n \in\{0, \ldots, 7\}$, as discussed in section 2.2, which constrains the allowed values of $N_{f}$ and $N_{c}$ via the equation

$$
2 N_{f} N_{c}=2 n
$$

We have assumed a single SQCD node of $H_{i}$ type. Due to the mod 3 condition for $U(1)$ nodes in equation (2.1), SQCD extensions of the non-Madrid embedding must have $N_{c}$ which is a not a multiple of 3 , as must any stringy hidden sector with an SQCD node attached to a visible sector $U(1)$ node. It is also possible to write down the allowed values of $N_{f}$ and $N_{c}$ for extensions of higher-node MSSM quivers. There are allowed values of $N_{f}$ and $N_{c}$ which break supersymmetry via the ADS superpotential.

Finally, for SQCD supersymmetry breaking with messenger flavors it is possible that the messengers fill out non-trivial standard model representations, in which case the ADS superpotential Higgses $G_{M S S M}$. The only possibility for the messenger flavors to be standard model singlets is an in extension of the non-Madrid embedding with $q^{(m)}=0$. See section 4.2 for an example.

\section{Hidden Sector Flavors}

The other possibility is that the $U\left(N_{c}\right)$ gauge theory which breaks supersymmetry is realized on an $H_{I}$ type node, in which case the $N_{f}$ flavors cannot be messenger fields. In this case there is no constraint on the allowed values of $N_{f}$ and $N_{c}$ since the flavors are hidden and they are not required to cancel a T-charge. Hidden sector fields are not required to be quasichiral and therefore in this case it is possible to realize vanilla SQCD with vector-like flavors. However, such flavors do not have masses protected by symmetry and are very heavy at a generic point in the moduli space of a string compactification. If so, the flavors can be integrated out, giving pure glue SQCD at low energies which does not break supersymmetry.

In clusters with $q^{(m)}=0$, the hidden sector SQCD flavors could also be quasichiral bifundamentals with protected masses, giving rise to a scenario very similar to that of the messenger flavor case. However, compared to the messenger flavor case the structure superpotential couplings is different, according to table 1 , and the possibilities are not as constrained.

\footnotetext{
${ }^{22}$ In these constructions the non-gauge invariance of $Q \tilde{Q}$ is compensated for by the non-gauge invariance of a closed-string modulus appearing in the correction.
} 


\subsubsection{Breaking Supersymmetry via a Retrofitted Fayet Model}

In [49] a retrofitted Fayet model which broke supersymmetry was presented in a simple quiver. We remind the reader that a Fayet model generically contains a $U(1)$ symmetry with a non-zero Fayet-Iliopoulos term $\xi$ and some number of fields charged under the $U(1)$. Since the F-term and D-term equations cannot be simultaneously satisfied, supersymmetry is broken. Given the many $U(1)$ symmetries in our hidden sectors, it seems natural that this model of supersymmetry breaking could be realized.

We would like to realize the Fayet model without needing to specify a concrete spectrum or hypercharge embedding. There are typically many heavy anomalous $Z^{\prime}$ bosons in a given quiver, but as emphasized in [54] the corresponding D-term equations should not be imposed since the $Z^{\prime}$ bosons can be integrated out of the low energy theory. Therefore, successful Fayet models should utilize massless $U(1)$ symmetries. Fortunately, in hidden hypercharge quivers there is typically a light $Z^{\prime}$ corresponding to the gauge symmetry $U(1)_{Y, H}$, as discussed in section 3.2. We will study the possibility of a single cluster hidden sector, though the arguments we present can be trivially generalized to the case of multiple cluster hidden sectors. Given that the hypercharged stringy hidden sector has a single sector, we will rescale $U(1)_{Y, H}$ by $1 / q^{(m)}$ to give a symmetry $U(1)_{F}$ for simplicity. This allows the discussion to proceed without reference to the value of $q^{(m)}$.

Let us discuss how $U(1)_{F}$ can play a role in supersymmetry breaking. Recall from section 2.3 that it is

$$
U(1)_{F}=\sum_{\alpha} U(1)_{\alpha}
$$

where the sum is over all hidden sector nodes. The only fields charged under this symmetry are the messengers $\Phi_{+}^{I}$ and $\Phi_{-}^{J}$, which carry positive and negative $U(1)_{F}$ charge, respectively. We assume that the messengers have non-zero mass. From table 1, we see that couplings of type $\mathcal{O}_{M}^{i} \mathcal{O}_{H}^{j}$ are forbidden in perturbation theory, while couplings of type $\mathcal{O}_{V} \mathcal{O}_{M}$ and $\mathcal{O}_{V} \mathcal{O}_{M} \mathcal{O}_{H}$ are at least string suppressed if the visible sector contains the exact MSSM spectrum. These couplings are either absent or generated via instantons or couplings to singlets. In the case that they are present, each coupling has an additional prefactor, given for example by worldsheet instantons ${ }^{23}$ in type IIa, which in principle allows them to be very small. Call $\epsilon$ the parameter of the largest such coupling.

In the limit where couplings of messengers to visible and/or hidden sector fields are absent, or in the limit $\epsilon \rightarrow 0$, the superpotential takes the form

$$
W=W_{M S S M}+m_{I J} \Phi_{+}^{I} \Phi_{-}^{J},
$$

where the mass matrix is generated via instantons or couplings to singlets. The relevant

\footnotetext{
${ }^{23}$ We are careful here to utilize the worldsheet instanton prefactor as the small parameter, rather than the D-instanton prefactor. This is because the same D-instanton which generates the messenger mass $\mathcal{O}_{\text {mess }}$ will also generate couplings of the form $\mathcal{O}_{\text {mess }} \mathcal{O}_{H}$ for perturbative couplings $\mathcal{O}_{H}$. In this case the D-instanton suppression of the operators cannot be tuned independently, whereas it is possible that the worldsheet instanton prefactors of each coupling could be tuned independently. The validity of our results in a given setup depend, of course, on whether a small parameter $\epsilon$ exists.
} 
F-term and D-term equations are

$$
\begin{gathered}
F_{\Phi_{+}^{I}}=m_{I J} \Phi_{-}^{J}=0 \quad F_{\Phi_{-}^{J}}=m_{I J} \Phi_{+}^{I}=0 \\
D_{F}=\xi+g^{2}\left(\sum_{I}\left|\Phi_{+}^{I}\right|^{2}-\sum_{I}\left|\Phi_{-}^{I}\right|^{2}\right)=0 .
\end{gathered}
$$

Supersymmetry is broken since these conditions cannot be simultaneously satisfied for non-zero $\xi$.

It is possible that the theory exhibits has an R-symmetry, with the requirements $R_{+}+$ $R_{-}=2$ for the R-charges of the positively and negatively charged fields. If the R-symmetry exists, taking $\epsilon$ small but finite gives a small explicit R-breaking which modifies the F-terms in a way that will typically restore supersymmetry. The non-supersymmetric vacuum is metastable and is separated from the supersymmetric vacuum by a distance in field space which is proportional to an inverse power of $\epsilon$. These are essentially the arguments for metastability presented in [48], where a small explicit R-symmetry breaking parameter $\epsilon$ sets the distance to the supersymmetric vacuum, and thus the lifetime of the metastable state.

Let us discuss one caveat. As specified currently, we have utilized the massless $U(1)_{F}$ symmetry that exists for any quiver with $q^{(m)} \neq 0$ to realize a Fayet model of supersymmetry breaking, where the messenger fields play the crucial role in the model and must obtain vacuum expectation values. Since $q^{(m)} \neq 0$, the messengers necessarily carry hypercharge and their vacuum expectation value spontaneously breaks $U(1)_{Y}$. Additionally, in extensions of the Madrid embedding the messengers are doublets of $S U(2)_{L}$ and therefore spontaneously break it. There is a simple way to avoid spontaneous breaking of the standard model without losing the Fayet model, however. Construct a Fayet model as specified in an extension where the messengers are $S U(2)_{L}$ singlets, such as in an extension of a non-Madrid quiver. Keep the same spectrum, but set $q^{(m)}=0$ so that the messengers do not have hypercharge. Nevertheless, $U(1)_{F}$ is a good massless symmetry and the breaking of supersymmetry breaking is as above, but without breaking $G_{M S S M}$. Thus, we can avoid this issue in a subclass of models with $q^{(m)}=0$.

We emphasize that this is only one possibility of breaking supersymmetry, though it is common. In concrete quivers there may be additional D-terms corresponding to other massless $U(1)$ symmetries that one should impose, giving more complicated realizations of the Fayet model. There may also be completely different methods of breaking supersymmetry, in particular there are likely realizations of the O'Raifeartaigh and Polonyi quivers of [49]. Our main point in this section is simply that $U(1)_{F}$ exists in broad classes of quivers and can give a realization of the Fayet model. We will present a concrete example in section 4.2 .

\subsubsection{Possibilities for Mediation}

In a successful supergravity embedding one could explore the possibility of gravity mediation. Since we are studying the $M_{p l} \rightarrow \infty$ limit and a supersymmetry breaking hidden 
sector in our models is connected to the visible sector, it is natural to explore alternatives. Given the importance of messenger fields and D-instantons in our quivers, as well as the presence of heavy and light $Z^{\prime}$ gauge symmetries, SUSY breaking could naturally be communicated by a combination of gauge mediation, $Z^{\prime}$ mediation, and $\mathrm{D}$-instanton mediation.

\section{Gauge Mediation}

It is natural to consider gauge mediation [55], since there are always messenger fields. In section 3.4 we discussed three common possibilities for supersymmetry breaking in stringy hidden valleys, which we called messenger SQCD, hidden SQCD, and Fayet models. In messenger SQCD and the Fayet models, the "messenger fields" themselves play a role in supersymmetry breaking, and thus should not be identified with possible messenger fields used for gauge mediation ${ }^{24}$. However, hidden SQCD can break supersymmetry without utilizing the messenger fields. In hidden SQCD or any other scenario which breaks supersymmetry without utilizing messenger fields, it is possible to consider the messenger fields as messengers of gauge mediation.

Let us begin by considering the case where messengers are only added for the sake of string consistency. In this case, messengers are always trivial under $S U(3)_{Q C D}$ and therefore gluino masses are not generated at one loop. Messenger masses are protected by $U(1)_{V}$ symmetry and therefore have good reason to be light. This is in contrast to other models in the literature, where messengers are vector like with respect to all symmetries and therefore light messengers require a considerable fine-tuning.

In extensions of the Madrid embedding, messengers are charged under $S U(2)_{L}$ and will generate soft masses for the corresponding gauginos. If the supersymmetry breaking hidden sector is hypercharged, the messengers carry hypercharge and will generate a soft mass for the bino. In extensions of the non-Madrid embedding, messengers are singlets of $S U(3)_{Q C D} \times S U(2)_{L}$ but carry hypercharge if the SUSY breaking hidden sector is hypercharged, generating a bino soft mass. Without adding non-required messengers, other mechanisms must account for the soft masses of the gluinos and squarks.

If one is willing to abandon our guiding principle of only adding fields for the sake of string consistency, it is possible to add messengers transforming as $3+\overline{3}$ of $S U(3)_{Q C D}$, which can be realized as $(a, i)+(\bar{a}, \bar{i})$. If the $H_{i}$ node is a $U(1)$, they could also be chiral under $U(1)_{i}$. In extensions of the Madrid embedding, the messengers added for string consistency are $(1,2)_{Y}+(1,2)_{-Y}$, but can never fill out a $5+\overline{5}$ of $S U(5)$ with the added $3+\overline{3}$, since they are chiral under $U(1)_{b}$. The messenger masses of the doublets and triplets are generically different, and the gluino will be lighter than the bino and wino if the $3+\overline{3}$ are vector like under $U(1)_{i}$. In extensions of the non-Madrid embedding, it is possible to add both doublet and triplet messengers, possibly filling out a $5+\overline{5}$. If the messengers required for consistency carry hypercharge there will be additional contributions to the bino mass beyond those of minimal gauge mediation from the $5+\overline{5}$. If the required messengers do not

\footnotetext{
${ }^{24}$ Remember that a messenger field, for us, is a field which is a bifundamental of a visible sector and hidden sector node, regardless of interpretation in any concrete physical scenario.
} 
carry hypercharge, then they do not participate in gauge mediation. In conclusion, there is no realization of minimal gauge mediation which utilizes the required messengers.

\section{$Z^{\prime}$ Mediation}

Another possibility for mediation of supersymmetry breaking is via $Z^{\prime}$ gauge bosons, which has been discussed in both field theoretic [56-59] and string theoretic models [60, 61]. In [58] a class of models was proposed where the dominant mediation mechanism was via a $U(1)^{\prime}$ gauge interaction which charged fields in both the visible sector and SUSY breaking hidden sector. In particular, the absence of messengers ensured the absence of leading contributions from gauge mediation. This is not possible in a stringy hidden valley, since messengers are generic.

It is still important to consider whether $Z^{\prime}$ mediation is a viable possibility for the partial mediation of supersymmetry breaking. For a generic stringy hidden valley, there exists no ${ }^{25}$ massless $U(1)$ which charges both the visible sector and either messenger or hidden sector fields. In particular, though $U(1)_{Y, H}$ is massless for a wide class of quivers, it never charges the standard model fields. However, in any specific quiver it is possible that there are additional massless $U(1)$ 's and $Z^{\prime}$ mediation could be realized.

Since there does not generically exist a non-anomalous $U(1)$ which charges both visible sector and hidden sector fields, the only $U(1)$ symmetries which could give rise to $Z^{\prime}$ mediation for a generic stringy hidden valley are anomalous. For example, $U(1)_{V}$ charges both standard model fields and messengers, but it is always anomalous and $Z_{V}^{\prime}$ has a large Stückelberg mass. Mediation of supersymmetry breaking via heavy $Z^{\prime}$ bosons must be subleading unless its mass is fine tuned to a low scale.

\section{D-Instanton Mediation}

D-instantons can generate non-perturbative corrections to the superpotential which couple visible sector fields to hidden sector fields, i.e. $\mathcal{O}_{V} \mathcal{O}_{H}$ couplings. As emphasized in table 1, these couplings are forbidden in perturbation theory but can be generated with exponential suppression via D-instantons. In [62] it was suggested to that these non-perturbative corrections can mediate supersymmetry breaking, giving rise to so-called D-instanton mediation. Though the original work only considered couplings of the form $\mathcal{O}_{V} \mathcal{O}_{H}$, it is also possible to consider D-instanton mediation via couplings of the form $\mathcal{O}_{M} \mathcal{O}_{H}$ and $\mathcal{O}_{V} \mathcal{O}_{M} \mathcal{O}_{H}$ which involve the messenger fields. These couplings are forbidden and string suppressed in perturbation theory, respectively. In certain quivers, some of these couplings are necessarily generated. For example, the messenger mass terms must be obtained via D-instantons or coupling to singlets, and in the former case an instanton which generates a messenger mass term $M \tilde{M}$ will also generate a coupling of the form $M \tilde{M} \mathcal{O}_{H}$ for any perturbative hidden sector coupling $\mathcal{O}_{H}$. However, the precise structure of the mediation is very model-dependent.

\footnotetext{
${ }^{25}$ With the exception of hypercharge, which could charge the messengers.
} 


\subsection{Implications of $Z^{\prime}$ Masses for Low Energy Physics}

Having discussed the importance of $Z^{\prime}$ bosons for the structure of stringy hidden valleys, including possibilities for dark matter and supersymmetry breaking, let us briefly comment on the implications of their masses for low energy physics.

As discussed, light $Z^{\prime}$ bosons obtain a mass via the standard Higgs mechanism, and could be $\mathcal{O}(\mathrm{TeV})$. Consider heavy $Z^{\prime}$ bosons. If arising from a string compactification they have a string scale Stückelberg mass, which could be fine-tuned to low scales, but is typically near the GUT or Planck scale. This is an important consideration when discussing the low energy physics of gauge theories with anomalous $Z^{\prime}$ bosons. If the $Z^{\prime}$ considered purely in an effective field theory where anomalous $U(1)$ symmetries require the presence of Chern-Simons terms, the $Z^{\prime}$ mass is a parameter. Regardless of its origin, it is useful to discuss what occurs at low energies as the $Z^{\prime}$ mass parameter is gradually lowered from Planck scale to weak scale.

For example, it is interesting to consider the mass $m_{V}$ of the heavy $Z_{V}^{\prime}$ gauge boson. This field always couples to both messenger fields $\psi$ and some standard model fermions $f$. If $m_{V}$ is Planck scale, it serves as a barrier between the visible and hidden sectors by suppressing the cross sections of processes such as $\psi \bar{\psi} \rightarrow Z_{V}^{\prime} \rightarrow f \bar{f}$ or $\psi \bar{\psi} \rightarrow Z_{V}^{\prime} \rightarrow Z \gamma$, making it unlikely that the dark matter scenario of [43] can account for the supposed $\gamma$-line [38] coming from the center of the galaxy. As $m_{V}$ is lowered, so is the $Z_{V}^{\prime}$ barrier and these annihilation processes give rise to interesting signals.

\subsection{Generality of Results and Higher-Node Extensions}

Though some of our discussion has focused on extensions of three-node MSSM quivers, our results are much more general.

Generically, most of the physics we have discussed throughout this section requires only a hidden sector with messengers carrying some non-zero $T_{V}$ charge. This occurs when the MSSM fields carry some net $T_{V}$ charge which must be canceled. This is true of nearly any stringy hidden valley of the type we study, regardless of the number of nodes in the MSSM quiver which has been extended. The only possible loophole is if all of the T-charge conditions (2.1) are satisfied but the masslessness conditions (2.2) are not, though the conclusions may hold in this case as well.

We can be more specific. Consider the three-node Madrid embedding. The salient feature which governs all extensions of the Madrid embedding is that hidden sectors are connected to the visible sector via messengers ending on the $U(2)_{b}$ node, since $T_{b} \neq 0$ for all three node quivers with the Madrid embedding. Therefore, hidden sectors added to threenode Madrid quivers can be added to any higher node quiver with the same $T_{b}$ charge. This is actually a very common possibility. To realize the MSSM gauge group at low energies, an arbitrary higher node MSSM quiver has gauge group $U(3)_{a} \times U(2)_{b} \times \prod_{i} U(1)_{c_{i}}$. Consider the hypercharge embedding

$$
U(1)_{Y}=\frac{1}{6} U(1)_{a}+\sum_{i} q_{i} U(1)_{c_{i}}
$$


and possible MSSM realizations. $Q, L, H_{u}$, and $H_{d}$ must transform under the $U(2)_{b}$ node, and $e^{c}, u^{c}$ and $d^{c}$ must not. The possible $T_{b}$ charges for an MSSM quiver are again $T_{b}= \pm 2 n$ with $n \in\{0, \ldots, 7\}$, and therefore the hidden sectors added to three-node Madrid quivers can be added to many higher node quivers, including the higher node Madrid embeddings with $q_{i}=\frac{1}{2} \forall i$. Such a quiver may require other additions to satisfy T-charge or M-charge conditions on other nodes, which is not necessary in the three-node case. Similar statements can be made about the non-Madrid embedding.

\section{Example Quivers}

In section 3 we discussed many aspects of physics which are generic in stringy hidden valleys, or at least a broad subclass, and discussed implications for low energy physics. We discussed the implications of $U(1)_{V}$ charged messengers for superpotential couplings, the ubiquity of both heavy and light $Z^{\prime}$ bosons, and axionic couplings required for anomaly cancellation. These ingredients had strong implications for both dark matter and supersymmetry breaking in these models.

In this section, we only seek to exemplify the more generic discussions of previous sections in concrete quivers with interesting low energy physics. We will discuss three extensions of Madrid quivers and two extensions of non-Madrid quivers.

\subsection{Hidden Extensions of the Madrid Embedding}

Let us study simple extensions of three-node MSSM quivers in the Madrid embedding. Before adding hidden sectors, the possible T-charges and M-charges of Madrid quivers with the exact MSSM spectrum are given in equation (2.8). Exploiting a quiver symmetry, we can take $T_{b}>0$ without loss of generality ${ }^{26}$, so that messengers added for consistency must end on the $U(2)_{b}$ node and be negatively charged under the $U(1)_{b}$.

\subsubsection{Dark Matter Annihilation into Photons via an Intermediate $Z^{\prime}$}

In this section we will demonstrate possibilities for dark matter and light $Z^{\prime}$ bosons discussed in section 3. The stringy hidden valley is demonstrated in figure 6 and has three hidden sector nodes, two of $H_{i}$ type and one of $H_{I}$ type. This hidden sector has $T_{b}=-6$ and can therefore be added consistently to any three-node MSSM quiver in the Madrid embedding with $T_{b}=6$. Since there is only a single hidden sector, we define $q \equiv q^{(m)} \neq 0$ and the messengers $M_{j}$ and $\tilde{M}_{k}$ have hypercharge $\pm q$, respectively. We take $q \in \mathbb{Z}+\frac{1}{2}$ in order to messengers particles with fractional electric charge. Messenger masses are protected by symmetry but can be generated non-perturbatively by instantons or via a perturbative coupling $S_{21} M_{j} \tilde{M}_{k}$ if $S_{21}$ obtains a vacuum expectation value. There is a perturbative superpotential term $S_{13} S_{32} S_{21}$ corresponding to the closed hidden sector loop.

\footnotetext{
${ }^{26}$ From the D-brane perspective, we can choose a brane on either $\pi_{b}$ or $\pi_{b}{ }^{\prime}$ to be the orientifold-image brane. This redundancy gives the mentioned quiver symmetry, so that there is a physically equivalent quiver with $T_{b}<0$ for every quiver with $T_{b}>0$ via mapping $b \leftrightarrow \bar{b}$.
} 


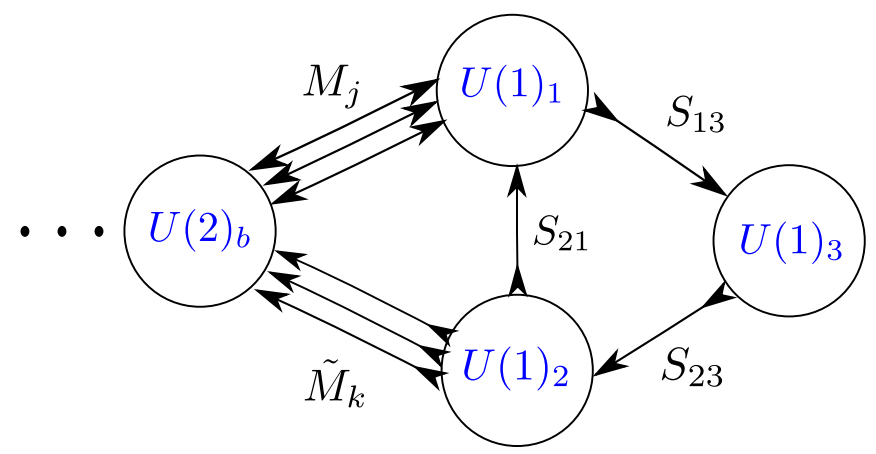

Figure 6. An example quiver which allows for dark matter annihilation into a photon via a $Z^{\prime} Z \gamma$ vertex. $U(1)_{1}+U(1)_{2}+U(1)_{3}$ is a massless $U(1)$, giving a light $Z^{\prime}$ boson.

We have constructed this quiver to exemplify some of the generic discussion of $Z^{\prime}$ physics in section 3.2. The linear combination ${ }^{27}$

$$
U(1)_{F}=U(1)_{1}+U(1)_{2}+U(1)_{3}
$$

is massless and gives a light $Z^{\prime}$ boson, as expected for hidden hypercharge quiver which extends a three-node MSSM quiver. Since there is a chiral excess of messengers on both $U(1)_{1}$ and $U(1)_{2}$, the associated boson $Z_{1}^{\prime}$ and $Z_{2}^{\prime}$ obtain a Stückelberg mass, as can be verified by explicitly checking equations $(2.2)$ for each $U(1)$. This is in agreement with the fact that there are $U(1)_{1} Y Y$ and $U(1)_{2} Y Y$ anomalies, due to the messenger fields.

The hidden sector contains three singlet fields which could be dark matter candidates. Any mass term in these fields is forbidden in perturbation theory, but can be generated by instantons at a suppressed scale, perhaps giving $\mathcal{O}(\mathrm{GeV})$ or $\mathcal{O}(\mathrm{TeV})$ dark matter. The $U(1)_{1} Y Y$ and $U(1)_{2} Y Y$ anomalies are canceled via the introduction of Chern-Simons terms. The necessary terms generate vertices $Z_{1}^{\prime} Z_{\gamma}$ and $Z_{2}^{\prime} Z_{\gamma}$ in the low energy theory and dark matter can annihilate into $Z \gamma$ via intermediate $Z_{1,2}^{\prime}$ bosons, as suggested in the dark matter scenario of $[42,43]$ to explain the tentative $\gamma$ line [38]. As emphasized in section 3 , though, in string compactifications these $Z^{\prime}$ bosons have a string scale Stückelberg mass within a few orders of $M_{p l}$, as opposed to the weak scale $Z^{\prime}$ of [43].

Dark matter realized as the neutral component of the messengers is also a possibility in this scenario. The messengers are the only particles charged under the non-anomalous symmetry $U(1)_{F}$, and therefore are stable. It can annihilate via $Z_{1,2}^{\prime} Z \gamma$ vertices into photons, but can also annihilate via a $Z_{b}^{\prime} Z \gamma$ vertex since there is a $U(1)_{b} Y Y$ anomaly. This dark matter candidate has a protected mass and weak interactions.

In addition, axionic couplings $\phi_{b} F_{Y} \wedge F_{Y}$ and $\phi_{b} F_{i} \wedge F_{i}$ are required for anomaly cancellation, where $F_{i}$ with $i=1,2$ are the field strengths of $U(1)_{1}$ and $U(1)_{2}$. Couplings of this form were used in a non-abelian gauge theory in [44] to give dark matter annihilation processes with photons in the final state, and it would be interesting to study their implications in this abelian model.

\footnotetext{
${ }^{27}$ Recall $U(1)_{F}=U(1)_{Y, H} / q^{(m)}$.
} 


\subsubsection{Many Light $Z^{\prime}$ Bosons}

In this subsection we will exemplify the possibility of many light $Z^{\prime}$ bosons as discussed in generality section 3.2. We study a two-cluster hidden sector, with quiver extension given in figure 7 which can be added consistently to any three-node MSSM quiver in the Madrid

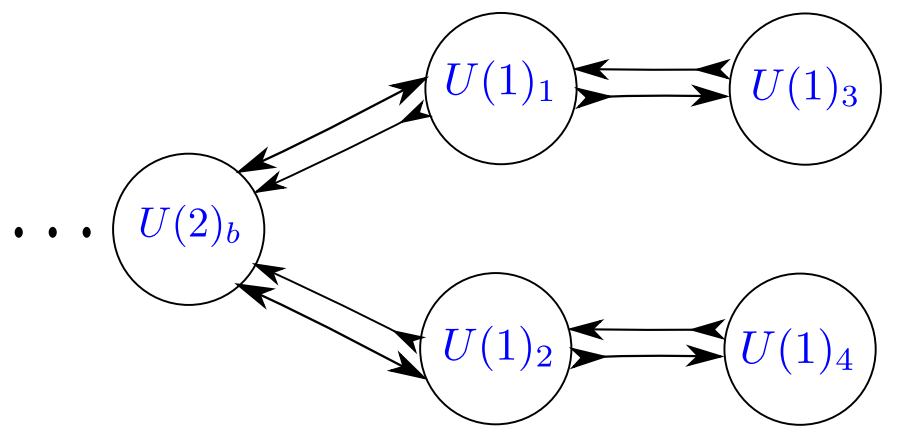

Figure 7. Quiver realizing two light $Z^{\prime}$ bosons.

embedding with $T_{b}=4$. The contribution of the hidden clusters to the hypercharge embedding are determined by two numbers $q^{(1)}$ and $q^{(2)}$, which must be non-zero for the messengers to have integral electric charge. As with any hidden hypercharge quiver extending a three-node MSSM quiver, $U(1)_{Y, H}$ satisfies the equations (2.2), which here is given by

$$
\begin{aligned}
U(1)_{Y, H} & =q^{(1)}\left[U(1)_{1}^{(1)}+U(1)_{2}^{(1)}\right]+q^{(2)}\left[U(1)_{1}^{(2)}+U(1)_{2}^{(2)}\right] \\
& \equiv U(1)_{Y, H}^{(1)}+U(1)_{Y, H}^{(2)}
\end{aligned}
$$

and the boson $Z_{Y, H}^{\prime}$ is light. From the general discussion of section 3.2, the symmetries $U(1)_{Y, H}^{(1)}$ and $U(1)_{Y, H}^{(2)}$ are also massless, since there are two hidden clusters with no chiral excess of messengers on $H_{i}$-type nodes. It can be explicitly checked that they satisfy the equations (2.2). Though it is non-generic, in this example all hidden sector $U(1)$ 's are massless.

\subsubsection{Dark Matter Annihilation and Axionic Couplings}

Here we show a simple implementation of the dark matter scenario resembling that of [44]. The quiver is shown in figure 8 . It contributes a $T_{b}$ charge of -6 , and therefore any quiver formed from this hidden sector and a Madrid quiver with $T_{b}=6$ is consistent. In order to obtain the appropriate $U(1)$ charges for the model, we take $U(1)_{X}=U(1)_{3}+$ $U(1)_{4}-U(1)_{1}-U(1)_{2}$ and assume that it is the lightest of any hidden sector $Z^{\prime}$ s. We find this assumption plausible since any of the individual $U(1)_{I}$ symmetries is anomalous. The quark mass terms $p \tilde{p}$ and $q \tilde{q}$ could arise via couplings to the singlets $r$ and $s$ when those fields obtain vacuum expectation values.

Consider the quark representations given in table 2. The dark matter candidates are the hidden sector pions $\pi_{H}^{ \pm}, \pi_{H}^{0}$ arising from the hidden sector quarks $p, \tilde{p}, q$, and $\tilde{q}$. To ensure the absence of fractionally charged massive particles, we must have $q^{(m)} \neq 0$. 
There are $U(1)_{b} Y Y$ and $U(1)_{b} S U(3)_{H}^{2}$ anomalies and cancellation via the Green-Schwarz mechanism requires the presence of couplings $\phi_{b} F_{Y} \wedge F_{Y}$ and $\phi_{b} \operatorname{Tr}(G \wedge G)$ where $F_{Y}$ is the hypercharge field strength and $G$ is the field strength of the hidden $S U(3)_{H}$. Hidden sector $U(1)_{X}$ charged pions can annihilate to $\pi_{H}^{0}$, which can then decay into photons via the axionic couplings. This is very similar to the setup in [44], to which we refer the reader for more details. One could also consider the possibility of messenger dark matter annihilation to $\gamma \gamma$ or $Z \gamma$ via an intermediate $Z_{b}^{\prime}$ boson.

\begin{tabular}{c|c|c} 
Field & $U(3)_{H}$ & $U(1)_{X}$ \\
\hline$p$ & $\square$ & +1 \\
$\tilde{p}$ & $\bar{\square}$ & -1 \\
$q$ & $\square$ & -1 \\
$\tilde{q}$ & $\bar{\square}$ & +1
\end{tabular}

Table 2. Representations of hidden sector quarks which form the hidden sector pions $\pi_{H}^{ \pm}$and $\pi_{H}^{0}$.

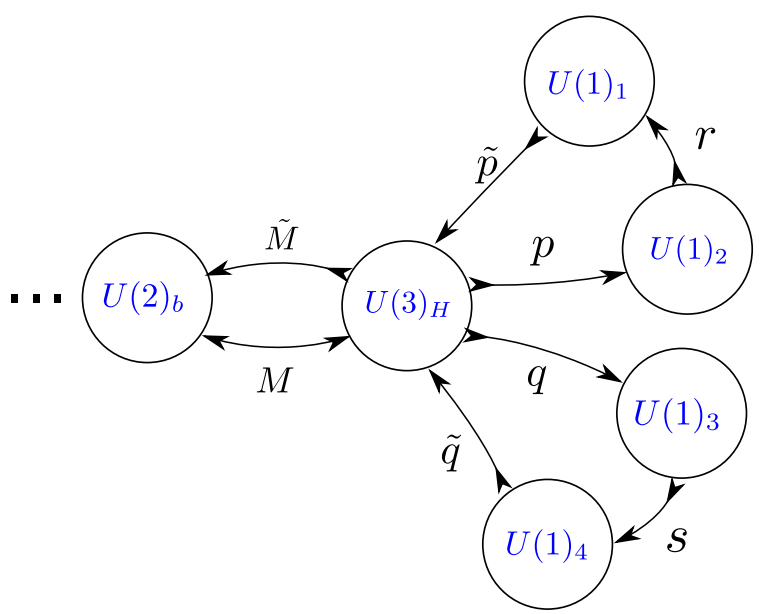

Figure 8. A quiver similar to that of [44]. The quark mass terms $p \tilde{p}$ and $q \tilde{q}$ arise via couplings to singlets $r$ and $s$.

This quiver is a bit contrived compared to some simpler possible realizations of [44]. We have two reasons for this. First, we have intentionally added many $U(1)_{I}$ nodes to forbid perturbative mass terms for hidden sector quarks, which would be string scale at a generic point in the moduli space of a string compactification. In this case the quark masses are typically high above the confinement scale $\Lambda_{H}$ and hidden sector pions do not form. Second, we have chosen the quiver such that the axionic couplings which give rise to $\pi_{H}^{0}$ decays are required, rather than put in by hand. This is due to the structure of $S U(2)_{L}$ charged messengers which also transform under $U(3)_{H}$, but are distinct from the hidden sector pions. Simpler realizations may also exist.

\subsection{Hidden Extensions of the Non-Madrid Embedding}

Let us turn to stringy hidden valleys which extend three-node MSSM quivers with the nonMadrid embedding. Before adding hidden sectors, the possible T-charges and M-charges 


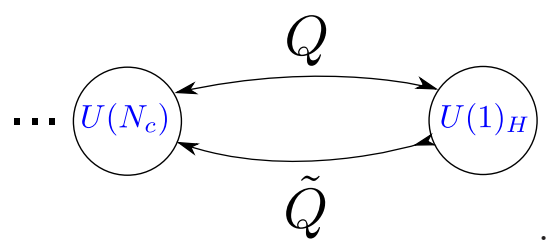

Figure 9. A simple single-node hidden sector which can realize the Fayet model of supersymmetry breaking. The mass term $Q \tilde{Q}$ is protected by symmetry but can be generated non-perturbatively.

of non-Madrid quivers with the exact MSSM spectrum are given in equation (2.9). Here messenger fields added for consistency must end on the $U(1)_{c}$ node.

Extensions of the non-Madrid embedding allow for a wider variety of possibilities than Madrid extensions. Let us state some of the differences. Since the messengers are not charged under $S U(2)_{L}$, it is possible to avoid fractionally charged massive particles by having a hidden sector which is not hypercharged. In such a case the messengers are MSSM singlets and any realization of supersymmetry breaking involving messenger VEVs doesn't automatically break $G_{\mathrm{MSSM}}$. Hidden sectors which are not hypercharged (i.e. $q_{i}=q_{I}=0$ for all $i, I$ ) are more natural in non-Madrid extensions, as discussed in section 2.4. In such a case symmetry and antisymmetry tensor representations can give rise to hidden sector fields, and all four bifundamental representations are allowed, instead of just two.

\subsubsection{Breaking SUSY with a Fayet Model}

Let us consider the simplest possible abelian hidden sector as an example, consisting of one node $U(1)_{H}$. Since we are considering extensions of MSSM quivers with $T_{c}>0$, we can add $N_{f}$ messenger fields of both types $(\bar{c}, H)$ and $(\bar{c}, \bar{H})$ that we will call $Q_{i}$ and $\tilde{Q}_{i}$, respectively. See figure 9 . Together these messenger have $T_{c}=-N_{f}$ and therefore can be added to MSSM quivers with $T_{c}=N_{f} \bmod 3$. For simplicity, we take $N_{f}=2$. We can take $U(1)_{Y, H}$ to be trivial without introducing fractionally charged massive particles, so that

$$
U(1)_{Y}=-\frac{1}{3} U(1)_{a}-\frac{1}{2} U(1)_{b}
$$

Thus, the MSSM spectrum is augmented only by a pair of MSSM $\operatorname{singlets} Q$ and $\tilde{Q}$. It is easy to see that $U(1)_{H}$ meets the conditions (2.2) and therefore gives a light $Z^{\prime}$ boson coupling only to $Q$ and $\tilde{Q}$. In this quiver $U(1)_{H}$ is the more generic $U(1)_{F}$ described in section 3.4.2, and it is therefore natural to expect that this realizes the Fayet model.

Let's discuss the superpotential. As usual, the messenger mass term $Q \tilde{Q}$ is forbidden by the symmetry $U(1)_{V}$, which here is $U(1)_{c}$. Phenomenology requires that some mechanism, such as a D-instanton or couplings to singlets with VEV, gives rise to a mass term $m Q \tilde{Q}$. The superpotential has the generic form

$$
W=\mathcal{O}_{V}+\epsilon \mathcal{O}_{V} Q \tilde{Q}+m Q \tilde{Q}
$$

where a sum over all couplings of type $\mathcal{O}_{V}$ is implied and we have considered only messenger sector couplings of the form $Q \tilde{Q}$ and not the irrelevant couplings $(Q \tilde{Q})^{k}$ for $k>2$. As 


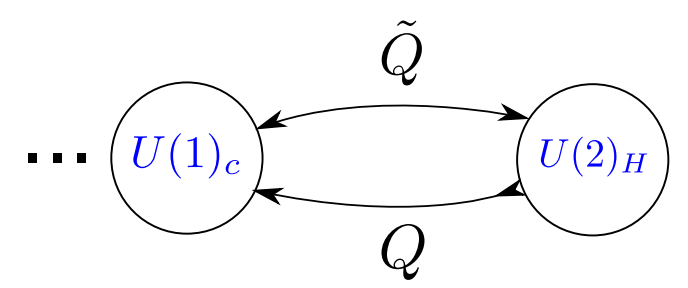

Figure 10. A simple realization of supersymmetry breaking via SQCD with messenger flavors.

discussed in section 3.4.2, we assume that couplings of the form $\mathcal{O}_{V} \mathcal{O}_{M}$ can be made parametrically small by tuning a parameter $\epsilon$. Then in the $\epsilon \rightarrow 0$ limit the superpotential is

$$
W=\mathcal{O}_{V}+m Q \tilde{Q}
$$

and the theory could have an R-symmetry depending on the structure of couplings $\mathcal{O}_{V}$. Supersymmetry requires $F_{Q}=m \tilde{Q}=0$ and $F_{\tilde{Q}}=m Q=0$, but since $U(1)_{H}$ is light and the messengers have $U(1)_{H}$ charge we must also impose the D-term constraint

$$
D=-\xi+g^{2}\left(|Q|^{2}-|\tilde{Q}|^{2}\right)=0,
$$

where $\xi$ is a non-zero Fayet-Iliopoulos term and $g$ is the gauge coupling. The scalar potential then takes the form

$$
V=m^{2}\left(Q^{2}+\tilde{Q}^{2}\right)+\frac{1}{2}\left[-\xi+g^{2}\left(Q^{2}-\tilde{Q}^{2}\right)\right]^{2}
$$

and supersymmetry is broken for generic $\xi$ since the D-term and F-term constraints cannot be simultaneously satisfied, giving $V>0$. If there is an R-symmetry, taking $\epsilon$ finite but small generically introduces a small explicit breaking ${ }^{28}$, restoring supersymmetry with a supersymmetric minimum at a distance $\sim 1 / \epsilon$ in field space. The non-supersymmetric vacuum is metastable for finite $\epsilon$, perhaps slightly shifted.

\subsubsection{Breaking SUSY with SQCD}

In this section we present a quiver where supersymmetric QCD with messenger flavors breaks supersymmetry as discussed in generality in section 3.4. For a concrete example let us take a $U\left(N_{c}\right)$ node with $N_{c}=2$ that we call $H$. We take a single flavor, so we are in the $N_{f}<N_{c}$ regime of SQCD. See figure 10. Messengers are attached to the $c$ node and the representations are $Q \sim\left(\bar{c}, N_{c}\right)$ and $\tilde{Q} \sim\left(\bar{c}, \bar{N}_{c}\right)$. These are the quarks of SQCD. These additions will contribute $T_{c}=-4$ and therefore this quiver can be added to any non-Madrid quiver with $T_{c}=4 \bmod 3=1 \bmod 3 . U(3 N)$ will never cancel $T_{c}$ charge.

Though $Q \tilde{Q}$ carries anomalous $U(1)$ charge, it is nevertheless possible [53] to generate the ADS superpotential. In addition, the fact that the quarks carry $U(1)_{c}$ charge does not influence the SQCD gauge dynamics, since $U(1)_{c}$ is anomalous and affects the low energy effective action only through global selection rules which protect the flavor mass.

\footnotetext{
${ }^{28}$ For example, if $\tilde{\mathcal{O}}_{V}$ is a particular coupling present in perturbation theory and the superpotential has a term $\epsilon \tilde{\mathcal{O}}_{V} Q \tilde{Q}$, finite but small $\epsilon$ gives a small explicit R-symmetry breaking.
} 
We assume that flavors obtain a small mass via instantons or couplings to singlets and that $\mathcal{O}_{V} \mathcal{O}_{M}$ couplings can be made small by a parameter $\epsilon$, perhaps given by a worldsheet instanton prefactor. At the very least, they are highly suppressed as in table 1. The superpotential is given by

$$
W=\mathcal{O}_{V}+\epsilon \mathcal{O}_{V} \mathcal{O}_{M}+\lambda_{1} \frac{\Lambda_{2}^{5}}{\tilde{Q} Q}+\lambda_{2} \tilde{Q} Q .
$$

In the $\epsilon \rightarrow 0$ and $\lambda_{2} \rightarrow 0$ limit, the ADS superpotential breaks supersymmetry but the potential exhibits runaway behavior. Since $Q \tilde{Q}$ carries anomalous $U(1)$ charge, it is plausible that there is an instanton which can generate the messenger mass but is unrelated to the non-perturbative effect which generates the ADS superpotential. This effect would make $\lambda_{2}$ finite and lift the runaway.

\section{Conclusions}

In this paper we have studied stringy hidden valley models. Broadly, these are hidden valleys where the quasi-hidden sectors are added to the MSSM for the sake of string consistency conditions which otherwise would not be satisfied. Since it is notoriously difficult to differentiate between quantum field theories and string compactifications at low energies, leveraging string consistency conditions to motivate extensions of the standard model is an interesting and promising approach.

We have focused on a subclass of these models heavily motivated by weakly coupled type II orientifold compactifications and their duals. We have shown that there are aspects of low energy physics which are generic in these stringy hidden valleys but are non-generic for an arbitrary hidden valley. In our models, this is primarily due to the fact that hidden sectors added for the sake of string consistency have messenger fields which are chiral under a symmetry $U(1)_{V}$ in the visible sector. The consequences are immediate and generic:

- Messenger masses are protected by symmetry but can be generated at a low scale by non-perturbative effects (e.g. D-instantons) or couplings to singlets.

- Other couplings are highly affected by $U(1)_{V}$ symmetry. For example, couplings of messengers to hidden sector fields are forbidden in perturbation theory. See table 1.

- There are $U(1)_{V} U(1)_{i} U(1)_{i}$ anomalies which are canceled via the Green-Schwarz mechanism through the introduction of Chern-Simons terms of the form $\phi_{V} F_{i} \wedge F_{i}$ and $B_{V} \wedge F_{V}$. The latter gives a Stückelberg mass to the $U(1)_{V}$ gauge boson.

- If there is a non-abelian node on which messengers end, there is a $U(1)_{V} S U\left(N_{i}\right) S U\left(N_{i}\right)$ anomaly whose cancellation requires the introduction of a $\phi_{V} \operatorname{Tr}\left(G_{i} \wedge G_{i}\right)$ term.

- The annihilation cross section of messengers into standard model fields is suppressed via the typically large mass of the anomalous $Z_{V}^{\prime}$ gauge boson. 
Heuristically, chirality of the messengers under $U(1)_{V}$ provides a coupling barrier between the visible and hidden sector. There is also a " $Z_{V}^{\prime}$ barrier" which prevents messenger annihilation into standard model fermions in proportion to the mass of $Z_{V}^{\prime}$.

The very broad subclass of stringy hidden valleys where the hidden sector is "hypercharged" exhibits other interesting features. Splitting the hypercharge embedding as

$$
U(1)_{Y}=U(1)_{Y, V}+U(1)_{Y, H}
$$

this subclass is defined as having a non-trivial contribution $U(1)_{Y, H}$. We call a quiver in this class a "hidden hypercharge quiver." Generic features include:

- Messengers carry hypercharge and contribute to the $U(1)_{V} Y Y$ anomaly coefficient. Unless precisely canceled by the contribution of the visible sector, the chiral spectrum exhibits a $U(1)_{V} Y Y$ anomaly. This is always the case for extensions of three-node MSSM quivers.

- Cancellation of the $U(1)_{V} Y Y$ anomaly requires the presence of a Chern-Simons term $\phi_{V} F_{Y} \wedge F_{Y}$. This gives rise to a $Z_{V}^{\prime} B B$ vertex which gives vertices involving $Z^{\prime}, \gamma$, and $Z$ after electroweak symmetry breaking.

- We require that any hidden hypercharge quiver must have hidden sector fields which are MSSM singlets. This requires that any non-zero contribution of a hidden sector node to the hypercharge must "propagate" to any connected hidden sector node. Thus, each node in a connected cluster contributes equally to the hypercharge embedding, so that the contribution of the $m^{\text {th }}$ cluster uniquely determined by a number $q^{(m)}$.

- Ensuring that fields in a cluster with $q^{(m)} \neq 0$ are MSSM singlets constrains the possible matter content to be two of the four possible bifundamental representations, while the other two are forbidden, along with symmetric and antisymmetric tensor representations.

- If $U(1)_{Y, V}$ is massless, then $U(1)_{Y, H}$ is also massless by the requirement that $U(1)_{Y}$ is massless. This is the case of any extension of a three-node MSSM quiver, and gives a light $Z_{Y, H}^{\prime}$ boson in the spectrum.

We see that this subclass is greatly simplified and that there are generically axionic couplings to the hypercharge field strength and usually an additional light $Z^{\prime}$ boson. These observations can have important phenomenological consequences.

We studied possibilities for dark matter and supersymmetry breaking in light of the rich structure of superpotential couplings, axionic couplings required for anomaly cancellation, and $Z^{\prime}$ physics. We considered possibilities for messenger dark matter and hidden sector dark matter. All possibilities are non-baryonic, since string consistency does not require the addition of messengers charged under $S U(3)_{Q C D}$. Messenger dark matter masses are protected by symmetry and could remain light. In the common case where $U(1)_{Y, H}$ is non-trivial and massless, this symmetry could protect messengers from decay, as could the 
many other $U(1)$ symmetries. Its annihilation cross section into standard model fermions via $Z_{V}^{\prime}$ is suppressed. Messenger dark matter in extensions of the Madrid embedding has weak interactions. Hidden sector dark matter candidates are standard model singlets and their masses are not necessarily protected by symmetry. The possibilities for messenger dark matter are more constrained than those of hidden sector dark matter. In hidden hypercharge quivers the presence of $U(1)_{V} Y Y$ anomalies requires $Z_{V}^{\prime} B B$ vertices and axionic couplings which can give dark matter annihilation processes with photons in the final state, as suggested in [42-44] to account for the tentative $\gamma$-ray at the Fermi LAT [38]. We emphasize that the annihilation cross section of processes involving an intermediate anomalous $Z^{\prime}$ is suppressed by the large $Z^{\prime}$ mass, would is typically near the GUT or Planck scale in a string compactification.

We also studied possibilities for the breaking of global supersymmetry via supersymmetric QCD or a retrofitted Fayet model. There are two natural possibilities for SQCD breaking: one where the SQCD flavors are messengers, and another where the flavors are hidden sector fields. In the case of messenger flavors, their flavor masses are protected by $U(1)_{V}$ symmetry and therefore it is plausible that they are light relative to the SQCD confinement scale. The ADS superpotential can be generated, despite the fact that $1 / Q \tilde{Q}$ carries anomalous $U(1)_{V}$ charge. Possible values of $N_{f}$ and $N_{c}$ are constrained by string consistency conditions. In the case of supersymmetry breaking with hidden sector flavors, the flavors can be vector-like, realizing standard SQCD. However, flavor masses are typically large and can be integrated out, giving supersymmetry preserving pure SQCD at low energies. It is also possible that the hidden sector flavors are chiral, in which case the SQCD theory is similar to the case of messenger flavors. Supersymmetry breaking via a Fayet model frequently appears in the limit where some parameters $\epsilon$ are small, due to the D-term constraint of the frequently massless symmetry $U(1)_{F}$. In the limit of finite $\epsilon$ the supersymmetry breaking vacuum becomes metastable. We find it interesting that there is a natural massless $U(1)$ for realizing the Fayet model. It is also likely that some stringy hidden valleys realize the retrofitted O'Raifeartaigh and Polonyi models of [49]. Natural possibilities for mediation include gauge mediation and D-instanton mediation. $Z^{\prime}$ mediation is also possible in a subclass of models, though it is not expected to be dominant. Minimal gauge mediation cannot be realized in a way which utilizes the messengers required for string consistency.

We also presented many example quivers realizing the possibilities discussed in the general section. We discussed three extensions of the Madrid embedding, which exhibited a $U(1)_{i} Y Y$ anomaly, multiple light $Z^{\prime}$ bosons, and possible dark matter annihilation processes with photons in the final state. In two extensions of the non-Madrid embedding we gave examples of supersymmetry breaking via SQCD and a Fayet model.

We leave many interesting possibilities for future work. One is to uncover other generic features in the class of models we have proposed. Another is to construct explicit models, performing a detailed study of new low energy signatures and studying experimental constraints on the allowed parameter space. It would also be interesting to study classes of gauge theories arising in other regions of the string landscape where string consistency conditions lead to concrete extensions of the standard model; that is, to find other classes 
of stringy hidden valleys.

\section{Acknowledgments}

We thank Shamit Kachru, Gordon Kane, Denis Klevers, Joseph Marsano, Mark Trodden, and Angel Uranga for useful conversations related to this work. J.H. thanks Matt Strassler for conversations on hidden valleys at TASI 2010 and Keith Dienes for conversations about dark matter. We are particularly grateful to Paul Langacker for useful conversations, comments on the manuscript, and recent collaborations. This work is supported in part by DOE grant DE-SC0007901. J.H. also acknowledges support from NSF grant PHY11-25915 and a DOE graduate fellowship. M.C. is also supported by the Fay R. and Eugene L. Langberg Endowed Chair and the Slovenian Research Agency (ARRS).

\section{References}

[1] M. J. Strassler and K. M. Zurek, Echoes of a hidden valley at hadron colliders, Phys.Lett. B651 (2007) 374-379, [hep-ph/0604261].

[2] B. Batell, S. Gori, and L.-T. Wang, Exploring the Higgs Portal with 10/Fb at the LHC, JHEP 1206 (2012) 172, [arXiv:1112.5180].

[3] J. Jaeckel and A. Ringwald, The Low-Energy Frontier of Particle Physics, Ann.Rev.Nucl.Part.Sci. 60 (2010) 405-437, [arXiv: 1002.0329].

[4] C. Cheung, G. Elor, L. J. Hall, and P. Kumar, Origins of Hidden Sector Dark Matter I: Cosmology, JHEP 1103 (2011) 042, [arXiv: 1010.0022].

[5] G. Cleaver, M. Cvetič, J. Espinosa, L. Everett, P. Langacker, et. al., Physics Implications of Flat Directions in Free Fermionic Superstring Models. 2. Renormalization Group Analysis, Phys.Rev. D59 (1999) 115003, [hep-ph/9811355].

[6] G. Cleaver, M. Cvetič, J. Espinosa, L. Everett, P. Langacker, et. al., Physics Implications of Flat Directions in Free Fermionic Superstring Models 1. Mass Spectrum and Couplings, Phys.Rev. D59 (1999) 055005, [hep-ph/9807479].

[7] G. Aldazabal, L. E. Ibanez, F. Quevedo, and A. M. Uranga, D-Branes at Singularities: a Bottom-Up Approach to the String Embedding of the Standard Model, JHEP 08 (2000) 002, [hep-th/0005067].

[8] H. Verlinde and M. Wijnholt, Building the Standard Model on a D3-Brane, JHEP 0701 (2007) 106, [hep-th/0508089].

[9] S. Krippendorf, M. J. Dolan, A. Maharana, and F. Quevedo, D-Branes at Toric Singularities: Model Building, Yukawa Couplings and Flavour Physics, JHEP 1006 (2010) 092, [arXiv: 1002.1790].

[10] R. Blumenhagen, M. Cvetič, and T. Weigand, Spacetime Instanton Corrections in $4 D$ String Vacua - the Seesaw Mechanism for D-Brane Models, Nucl. Phys. B771 (2007) 113-142, [hep-th/0609191].

[11] L. E. Ibanez and A. M. Uranga, Neutrino Majorana Masses from String Theory Instanton Effects, JHEP 03 (2007) 052, [hep-th/0609213]. 
[12] B. Florea, S. Kachru, J. McGreevy, and N. Saulina, Stringy Instantons and Quiver Gauge Theories, JHEP 05 (2007) 024, [hep-th/0610003].

[13] L. E. Ibanez and . Richter, Robert, Stringy Instantons and Yukawa Couplings in Mssm-Like Orientifold Models, JHEP 03 (2009) 090, [arXiv:0811.1583].

[14] P. Anastasopoulos, E. Kiritsis, and A. Lionetto, On Mass Hierarchies in Orientifold Vacua, JHEP 08 (2009) 026, [arXiv:0905.3044].

[15] M. Cvetič, J. Halverson, and . Richter, Robert, Realistic Yukawa Structures from Orientifold Compactifications, JHEP 12 (2009) 063, [arXiv: 0905.3379].

[16] M. Cvetič, J. Halverson, and R. Richter, Mass Hierarchies from MSSM Orientifold Compactifications, JHEP 07 (2010) 005, [arXiv: 0909.4292].

[17] M. Cvetič, J. Halverson, and R. Richter, Mass Hierarchies Vs. Proton Decay in MSSM Orientifold Compactifications, arXiv:0910.2239.

[18] P. Anastasopoulos and A. Lionetto, Quark Mass Hierarchies in D-Brane Realizations of the Standard Model, Fortsch.Phys. 58 (2010) 703-707, [arXiv:0912.0121].

[19] M. Cvetič and P. Langacker, D-Instanton Generated Dirac Neutrino Masses, Phys. Rev. D78 (2008) 066012, [arXiv:0803.2876].

[20] M. Cvetič, J. Halverson, P. Langacker, and R. Richter, The Weinberg Operator and a Lower String Scale in Orientifold Compactifications, JHEP 10 (2010) 094, [arXiv:1001.3148].

[21] M. Cvetič, J. Halverson, and P. Langacker, Singlet Extensions of the MSSM in the Quiver Landscape, JHEP 09 (2010) 076, [arXiv:1006.3341].

[22] F. Fucito, A. Lionetto, J. Morales, and R. Richter, Dynamical Supersymmetry Breaking in Intersecting Brane Models, JHEP 1011 (2010) 024, [arXiv:1007.5449].

[23] E. Kiritsis, M. Lennek, and B. Schellekens, SU(5) Orientifolds, Yukawa Couplings, Stringy Instantons and Proton Decay, Nucl.Phys. B829 (2010) 298-324, [arXiv:0909.0271].

[24] P. Anastasopoulos, G. K. Leontaris, R. Richter, and A. N. Schellekens, SU(5) D-Brane Realizations, Yukawa Couplings and Proton Stability, JHEP 12 (2010) 011, [arXiv: 1010.5188].

[25] D. Berenstein and S. Pinansky, The Minimal Quiver Standard Model, Phys. Rev. D75 (2007) 095009, [hep-th/0610104].

[26] M. Cvetič, J. Halverson, and P. Langacker, Implications of String Constraints for Exotic Matter and Z' S Beyond the Standard Model, JHEP 11 (2011) 058, [arXiv:1108.5187].

[27] L. A. Anchordoqui et. al., Z'-Gauge Bosons as Harbingers of Low Mass Strings, arXiv: 1107.4309

[28] P. Anastasopoulos, T. P. T. Dijkstra, E. Kiritsis, and A. N. Schellekens, Orientifolds, Hypercharge Embeddings and the Standard Model, Nucl. Phys. B759 (2006) 83-146, [hep-th/0605226].

[29] M. Cvetič and J. Halverson, TASI Lectures: Particle Physics from Perturbative and NonPerturbative Effects in D-Braneworlds, arXiv:1101.2907.

[30] P. Anastasopoulos, M. Bianchi, E. Dudas, and E. Kiritsis, Anomalies, Anomalous U(1)'s and Generalized Chern-Simons Terms, JHEP 11 (2006) 057, [hep-th/0605225].

[31] L. E. Ibanez, F. Marchesano, and R. Rabadan, Getting Just the Standard Model at 
Intersecting Branes, JHEP 11 (2001) 002, [hep-th/0105155].

[32] E. Witten, An SU(2) Anomaly, Phys. Lett. B117 (1982) 324-328.

[33] P. Langacker and G. Steigman, Requiem for an FCHAMP? Fractionally CHArged, Massive Particle, Phys.Rev. D84 (2011) 065040, [arXiv:1107.3131].

[34] S. Kachru and A.-K. Kashani-Poor, Moduli Potentials in Type Iia Compactifications with Rr and NS Flux, JHEP 0503 (2005) 066, [hep-th/0411279].

[35] P. G. Camara, A. Font, and L. Ibanez, Fluxes, Moduli Fixing and Mssm-Like Vacua in a Simple IIA Orientifold, JHEP 0509 (2005) 013, [hep-th/0506066].

[36] P. Langacker, The Physics of Heavy Z-Prime Gauge Bosons, Rev. Mod. Phys. 81 (2009) 1199-1228, [arXiv: 0801.1345].

[37] M. Cvetič, G. Shiu, and A. M. Uranga, Chiral Four-Dimensional $\mathcal{N}=1$ Supersymmetric Type IIA Orientifolds from Intersecting D6-Branes, Nucl. Phys. B615 (2001) 3-32, [hep-th/0107166].

[38] C. Weniger, A Tentative Gamma-Ray Line from Dark Matter Annihilation at the Fermi Large Area Telescope, JCAP 1208 (2012) 007, [arXiv:1204.2797].

[39] E. Tempel, A. Hektor, and M. Raidal, Fermi 130 GeV Gamma-Ray Excess and Dark Matter Annihilation in Sub-Haloes and in the Galactic Centre, arXiv:1205.1045.

[40] A. Boyarsky, D. Malyshev, and O. Ruchayskiy, Spectral and Spatial Variations of the Diffuse Gamma-Ray Background in the Vicinity of the Galactic Plane and Possible Nature of the Feature at $130 \mathrm{GeV}$, arXiv:1205.4700.

[41] M. Su and D. P. Finkbeiner, Strong Evidence for Gamma-Ray Line Emission from the Inner Galaxy, arXiv:1206.1616.

[42] E. Dudas, Y. Mambrini, S. Pokorski, and A. Romagnoni, (In)Visible Z' and Dark Matter, JHEP 08 (2009) 014, [arXiv: 0904.1745].

[43] E. Dudas, Y. Mambrini, S. Pokorski, and A. Romagnoni, Extra U(1) as Natural Source of a Monochromatic Gamma Ray Line, arXiv:1205.1520.

[44] J. Fan and M. Reece, A Simple Recipe for the 111 and 128 GeV Lines, arXiv:1209.1097.

[45] I. Affleck, M. Dine, and N. Seiberg, Dynamical Supersymmetry Breaking in Four-Dimensions and Its Phenomenological Implications, Nucl.Phys. B256 (1985) 557.

[46] I. Affleck, M. Dine, and N. Seiberg, Dynamical Supersymmetry Breaking in Supersymmetric QCD, Nucl.Phys. B241 (1984) 493-534.

[47] K. A. Intriligator, N. Seiberg, and D. Shih, Dynamical SUSY breaking in meta-stable vacua, JHEP 0604 (2006) 021, [hep-th/0602239].

[48] K. A. Intriligator, N. Seiberg, and D. Shih, Supersymmetry Breaking, R-Symmetry Breaking and Metastable Vacua, JHEP 0707 (2007) 017, [hep-th/0703281].

[49] O. Aharony, S. Kachru, and E. Silverstein, Simple Stringy Dynamical SUSY Breaking, Phys. Rev. D76 (2007) 126009, [arXiv:0708.0493].

[50] M. Cvetič and T. Weigand, Hierarchies from D-Brane Instantons in Globally Defined Calabi-Yau Orientifolds, Phys. Rev. Lett. 100 (2008) 251601, [arXiv:0711.0209].

[51] M. Cvetič and T. Weigand, A String Theoretic Model of Gauge Mediated Supersymmetry Beaking, arXiv:0807.3953. 
[52] K. A. Intriligator and N. Seiberg, Lectures on Supersymmetry Breaking, Class. Quant. Grav. 24 (2007) S741-S772, [hep-ph/0702069].

[53] M. Haack, D. Krefl, D. Lüst, A. Van Proeyen, and M. Zagermann, Gaugino Condensates and D-Terms from D\%-Branes, JHEP 01 (2007) 078, [hep-th/0609211].

[54] K. A. Intriligator and N. Seiberg, The Runaway Quiver, JHEP 0602 (2006) 031, [hep-th/0512347].

[55] G. Giudice and R. Rattazzi, Theories with Gauge Mediated Supersymmetry Breaking, Phys.Rept. 322 (1999) 419-499, [hep-ph/9801271].

[56] P. Binetruy and E. Dudas, Gaugino Condensation and the Anomalous U(1), Phys.Lett. B389 (1996) 503-509, [hep-th/9607172].

[57] G. Dvali and A. Pomarol, Anomalous U(1) as a Mediator of Supersymmetry Breaking, Phys.Rev.Lett. 77 (1996) 3728-3731, [hep-ph/9607383].

[58] P. Langacker, G. Paz, L.-T. Wang, and I. Yavin, Z'-Mediated Supersymmetry Breaking, Phys.Rev.Lett. 100 (2008) 041802, [arXiv:0710.1632].

[59] P. Langacker, G. Paz, L.-T. Wang, and I. Yavin, Aspects of Z-Prime - Mediated Supersymmetry Breaking, Phys.Rev. D77 (2008) 085033, [arXiv:0801.3693].

[60] H. Verlinde, L.-T. Wang, M. Wijnholt, and I. Yavin, A Higher Form (Of) Mediation, JHEP 0802 (2008) 082, [arXiv:0711.3214].

[61] T. W. Grimm and A. Klemm, U(1) Mediation of Flux Supersymmetry Breaking, JHEP 0810 (2008) 077, [arXiv: 0805.3361].

[62] M. Buican and S. Franco, SUSY Breaking Mediation by D-Brane Instantons, JHEP 12 (2008) 030, [arXiv: 0806.1964]. 